\title{
A Bright and Fast Red Fluorescent Protein Voltage Indicator That Reports Neuronal Activity in Organotypic Brain Slices
}

\author{
Ahmed S. Abdelfattah, ${ }^{1}$ @Samouil L. Farhi, ${ }^{2 *}$ Yongxin Zhao, ${ }^{1 *}$ Daan Brinks, ${ }^{2}$-Peng Zou, ${ }^{2}$ Araya Ruangkittisakul, ${ }^{3}$ \\ Jelena Platisa, ${ }^{4,5}$-Vincent A. Pieribone, ${ }^{4,5,6}$ Klaus Ballanyi, ${ }^{3}$ Adam E. Cohen, ${ }^{2,7,8}$ and Robert E. Campbell ${ }^{1}$ \\ ${ }^{1}$ Department of Chemistry, University of Alberta, Edmonton, Alberta T6G 2G2, Canada, ${ }^{2}$ Department of Chemistry and Chemical Biology, Harvard \\ University, Cambridge, Massachusetts 02138, ${ }^{3}$ Department of Physiology, University of Alberta, Edmonton, Alberta T6G 2H7, Canada, ${ }^{4}$ The John B. Pierce \\ Laboratory, Inc., New Haven, Connecticut 06519, ${ }^{5}$ Cellular and Molecular Physiology, Yale University School of Medicine, New Haven, Connecticut 06520, \\ ${ }^{6}$ Neurobiology, Yale University School of Medicine, New Haven, Connecticut 06510, ${ }^{7}$ Department of Physics, Harvard University, Cambridge, \\ Massachusetts 02138, and ${ }^{8}$ Howard Hughes Medical Institute, Harvard University, Cambridge, Massachusetts 02138
}

Optical imaging of voltage indicators based on green fluorescent proteins (FPs) or archaerhodopsin has emerged as a powerful approach for detecting the activity of many individual neurons with high spatial and temporal resolution. Relative to green FP-based voltage indicators, a bright red-shifted FP-based voltage indicator has the intrinsic advantages of lower phototoxicity, lower autofluorescent background, and compatibility with blue-light-excitable channelrhodopsins. Here, we report a bright red fluorescent voltage indicator (fluorescent indicator for voltage imaging red; FlicR1) with properties that are comparable to the best available green indicators. To develop FlicR1, we used directed protein evolution and rational engineering to screen libraries of thousands of variants. FlicR 1 faithfully reports single action potentials $(\sim 3 \%$ $\Delta F / F)$ and tracks electrically driven voltage oscillations at $100 \mathrm{~Hz}$ in dissociated Sprague Dawley rat hippocampal neurons in single trial recordings. Furthermore, FlicR1 can be easily imaged with wide-field fluorescence microscopy. We demonstrate that FlicR1 can be used in conjunction with a blue-shifted channelrhodopsin for all-optical electrophysiology, although blue light photoactivation of the FlicR1 chromophore presents a challenge for applications that require spatially overlapping yellow and blue excitation.

Key words: biosensors; fluorescence imaging; fluorescent proteins; genetically encoded indicators; protein engineering; voltage imaging

\section{Significance Statement}

Fluorescent-protein-based voltage indicators enable imaging of the electrical activity of many genetically targeted neurons with high spatial and temporal resolution. Here, we describe the engineering of a bright red fluorescent protein-based voltage indicator designated as FlicR1 (fluorescent indicator for voltage imaging red). FlicR1 has sufficient speed and sensitivity to report single action potentials and voltage fluctuations at frequencies up to $100 \mathrm{~Hz}$ in single-trial recordings with wide-field microscopy. Because it is excitable with yellow light, FlicR1 can be used in conjunction with blue-light-activated optogenetic actuators. However, spatially distinct patterns of optogenetic activation and voltage imaging are required to avoid fluorescence artifacts due to photoactivation of the FlicR1 chromophore.

\section{Introduction}

The human brain has $\sim 80$ billion neurons (Herculano-Houzel, 2012) that are organized into elaborate circuits that form the basis of sensory integration, motor coordination, and higher brain functions. Because these neuronal circuits are made up of numerous spatially dispersed neurons, simultaneous recording of activity from many locations is key to understanding how brain function emerges from these circuits (Pesaran et al., 2008; Cohen and Maunsell, 2009). Toward this goal, small-molecule organic dyes with fluorescent signals that change in response to changes in the membrane potential (Davila et al., 1973) or calcium ion $\left(\mathrm{Ca}^{2+}\right)$ concentration (Brown et al., 1975; Tsien, 1980) 
have been used over the past four decades to image neuronal activity. A drawback of organic dyes is that bath application typically leads to a summed signal from many stained cells and single cell resolution is lost unless the dye is microinjected. Genetically encoded indicators overcome this limitation because they can be targeted to a sparse subset of cells using cell-type-specific promoters.

Despite the fact that prototypes of both genetically encoded voltage and $\mathrm{Ca}^{2+}$ indicators were first reported in 1997 (Miyawaki et al., 1997; Siegel and Isacoff, 1997), the development of robust voltage indicators has lagged far behind $\mathrm{Ca}^{2+}$ indicators. The design that has proven most successful for developing $\mathrm{Ca}^{2+}$ indicators with large dynamic range is based on fusing a circularly permuted (cp) fluorescent protein (FP) (Baird et al., 1999) to a $\mathrm{Ca}^{2+}$-sensing domain (Nagai et al., 2001; Nakai et al., 2001; Zhao et al., 2011; Chen et al., 2013). Similarly designed voltage indicators, constructed by fusing FPs to voltage-sensing domains, typically suffer from small response amplitude, slow kinetics, and/or poor membrane localization (Siegel and Isacoff, 1997; Ataka and Pieribone, 2002). However, two recently reported green FPbased voltage indicators, ArcLight (Jin et al., 2012) and ASAP1 (St-Pierre et al., 2014), are able to resolve single action potentials in neurons in single trials.

Red-shifted fluorophores that require longer wavelength excitation light provide the added advantages of lower phototoxicity, deeper tissue penetration, and lower autofluorescent background (Shu et al., 2009). Moreover, they are spectrally distinct from green fluorescent indicators and blue-light-excitable channelrhodopsin variants (Alford et al., 2013). Unfortunately, the only reported attempt to engineer an indicator based on a cp red FP (cpRFP) resulted in indicators that are not practically useful due to small fluorescence responses ( $\sim 1 \% \Delta F / F$ to $200 \mathrm{mV}$ step potentials) and slow kinetics ( $\tau_{\text {on }} \sim 60 \mathrm{~ms}$ and $\tau_{\text {off }} \sim 150 \mathrm{~ms}$ ) (Gautam et al., 2009). We set out to develop a new FP-based voltage indicator that combined the advantages of red-shifted fluorescent emission with the higher brightness of FPs while also affording the fast kinetics required to report neuronal activity. Note that we follow the convention of referring to FPs that emit in the 590-620 nm range as "red," although such wavelengths are more accurately described as orange.

Here, we describe the development of a bright and fast red voltage indicator based on a fusion of a cpRFP (cpmApple) and the voltage-sensing domain (VSD) from a Ciona intestinalis (Ci) voltage-sensitive phosphatase (Murata et al., 2005). This fusion was used as a template for directed protein evolution to improve brightness and voltage sensitivity. The end product is FlicR1 (fluorescent indicator for voltage imaging red), with kinetics and relative response amplitude comparable to the best available green voltage indicators. We show that FlicR1 reports single action potentials in single-trial recordings from neurons and can track high-frequency voltage fluctuations (up to $100 \mathrm{~Hz}$ ). We also demonstrate that FlicR1 can be combined with a blue-shifted channelrhodopsin, PsChR (Govorunova et al., 2013), and spatially patterned blue illumination to simultaneously perturb and

was a generous gift from Michael W. Davidson. Humanized-PsChR-eYFP under CMV control in pcDNA3.1 backbone was generously provided by J. Spudich. pGEMTEZ-Kir2.1 was a gift from Richard Axel, Joseph Gogos, and C. Ron Yu (Addgene plasmid \#32641). The constructs described in this manuscript will be available on Addgene. A.E.C. is a founder of Q-State Biosciences. The remaining authors declare no competing financial interests.

*S.L.F. and Y.Z. contributed equally to this work.

Correspondence should be addressed to Robert E. Campbell, Department of Chemistry, University of Alberta, 11227 Saskatchewan Drive, Edmonton, Alberta T6G 2G2, Canada. E-mail: robert.e.campbell@ualberta.ca.

DOI:10.1523/JNEUROSCI.3484-15.2016

Copyright $\odot 2016$ the authors $\quad 0270-6474 / 16 / 362459-15 \$ 15.00 / 0$ image membrane potential in the same neuron. However, blue light photoactivation of the FlicR1 chromophore as observed in R-GECO1, which has the same FP barrel and chromophore (Wu et al., 2013), presents a challenge for applications that require spatially overlapping yellow and blue excitation.

\section{Materials and Methods}

Molecular biology to construct FlicR variants. PCR amplification was used to construct the DNA template for FlicR. Synthetic oligonucleotides (Integrated DNA Technologies) were used as primers for amplification and Pfu polymerase (Thermo Fisher Scientific) was used to maintain highfidelity DNA replication. Overlap PCR was used to link CiVSD to cpmApple FP. Random mutagenesis was performed with error-prone PCR amplification using Taq polymerase (New England Biolabs) in the presence of $\mathrm{MnCl}_{2}(0.1 \mathrm{~mm})$ and $800 \mu \mathrm{M}$ excess dTTP and dCTP. Randomization of targeted codons was performed with QuikChange Lightning kits (Agilent Technologies). Restriction endonucleases (Thermo Fisher Scientific) were used to digest PCR products and expression vectors. Agarose gel electrophoresis was used to purify DNA products from PCR and restriction digestion reactions. The DNA was extracted from the gels using the GeneJET gel extraction kit (Thermo Fisher Scientific). Ligations were performed using T4 DNA ligase (Thermo Fisher Scientific).

DNA encoding the first 242 aa from CiVSD (VSD242) was generated by PCR amplification of CiVSD domain from the voltage sensor VSFP3.1 (Lundby et al., 2008) using forward primer (FW-BamHI-VSD) and reverse primer (RV-cpmApple-VSD242). DNA encoding the cpmApple variant was generated by PCR amplification of gene encoding R-GECO1 using forward primer (FW-VSD242-cpmApple) and reverse primer (RV-XbaI-cpmApple). Primers RV-cpmApple-VSD242 and FW-VSD 242-cpmApple contain an overlap region that was used to join these two genes together by overlap PCR. Primers RV-cpmApple-VSD242 and FW-VSD242-cpmApple also contained two fully randomized codons (NNK codons) that link the two genes together, generating 1024 variants. The length of the VSD amplified by PCR was varied (VSD 236, VSD237, VSD238, VSD239, VSD240, and VSD241). Other sets of overlap primers, along with FW-BamHI-VSD primer and RV-XbaI-cpmApple primer, were used to link DNA encoding cpmApple to the shorter VSDs as outlined above for VSD242. This led to a library of 1024 FlicR variants for each length of VSD.

Error-prone PCR together with DNA shuffling were used to construct libraries in the following rounds of directed evolution on FlicR variants from VSD239 library. PCR products were purified by agarose gel electrophoresis, digested, and ligated into a modified pcDNA3.1(+) vector as described below.

Plasmid for dual E. coli and mammalian cell expression. A vector for expression in prokaryotic and eukaryotic systems was constructed based on mammalian expression vector pcDNA3.1 $(+)$. To facilitate prokaryotic expression, an E. coli ribosome-binding site (aggaggaa) for prokaryotic translation was introduced using a QuikChange reaction (Agilent Technologies). We named the resulting vector pcDuEx0.5. The transcription of the encoded genes relies on the weak activity of the cytomegalovirus (CMV) promoter in E. coli cells (Lewin et al., 2005). pcDuEx0.5 exhibited moderate expression of FlicR variants in E. coli cells and showed comparable expression levels in HeLa cells compared with the original pcDNA3.1 $(+)$. We used pcDuEx0.5 as the vector for screening of FlicR libraries. Other dual expression vectors have been developed previously (Mullinax et al., 2002).

Plasmid for neuronal expression. To express FlicR1 in neurons, FlicR1 was cloned from plasmid pcDuEx0.5 into the BamHI/HindIII sites of an AAV2 vector using FW-BamHI-VSD and RV-HindIII-cpmApple primers. FlicR1 expression was controlled using human synapsin I promoter to preferentially express in neurons. A 3' Woodchuck Hepatitis Virus Posttranscriptional Regulatory Element (WPRE) sequence was used to enhance expression. For channelrhodopsin expression, a TsChR-TSeGFP fusion was cloned into the same plasmid, where TS represents a KiR2.1-trafficking sequence used to improve membrane trafficking of channelrhodopsins. The amino acid sequence of the TS sequence is KSRITSEGEYIPLDQIDINV. ChR2(H134R)-eGFP was expressed from a 
lentiviral construct under a CaMKII promoter described previously (Hochbaum et al., 2014).

Screening of FlicR library variants in E. coli. Gene libraries of FlicR variants were transformed into electrocompetent E. coli strain DH10B (Invitrogen). E. coli cells were then plated and cultured at $37^{\circ} \mathrm{C}$ on LB agar plates supplemented with ampicillin $(400 \mu \mathrm{g} / \mathrm{ml})$ to obtain $500-$ 1000 colonies per plate. Colonies were then imaged using a custom imaging setup described previously (Cheng and Campbell, 2006). To screen for FlicR mutant brightness, plate images were acquired using a 560/40 nm excitation filter and 630/60 nm emission filter. For each round of random mutagenesis, $\sim 10,000$ colonies ( $10-20$ plates) were screened. For each library generated by codon randomization, approximately three times more colonies than the expected randomization library size were screened. Colonies with the top $0.01 \%$ fluorescence brightness were manually picked and cultured in $4 \mathrm{ml}$ of LB medium supplemented with ampicillin $(100 \mu \mathrm{g} / \mathrm{ml})$. Plasmids were then extracted using the GeneJET plasmid miniprep kit (Thermo Fisher Scientific).

Expression and in vitro spectroscopic characterization of FlicR1. To purify FlicR1, the pcDuEx0.5 plasmid harboring FlicR1 was used to transform electrocompetent $E$. coli $\mathrm{DH} 10 \mathrm{~B}$ cells (Invitrogen). The E. coli were grown on LB agar plates supplemented with ampicillin $(400 \mu \mathrm{g} / \mathrm{ml})$. A colony was used to inoculate $8 \mathrm{ml}$ of LB liquid medium $(100 \mu \mathrm{g} / \mathrm{ml}$ ampicillin) and grown overnight at $37^{\circ} \mathrm{C}$. The next day, the $8 \mathrm{ml}$ of bacterial culture was added to $500 \mathrm{ml}$ of LB medium $(100 \mu \mathrm{g} / \mathrm{ml}$ ampicillin) and grown for $4 \mathrm{~h}$ at $37^{\circ} \mathrm{C}$, and then grown for $48 \mathrm{~h}$ at $25^{\circ} \mathrm{C}$. The cell pellets were then collected by centrifugation and lysed by suspension in $\mathrm{B}-\mathrm{PER}$ solution (Pierce). The insoluble fraction (containing the membrane proteins) was collected after centrifugation and resuspended in a solution of $2 \% n$-dodecyl- $\beta$-D-maltopyranoside (Anatrace) in Trisbuffered saline. The suspension was then homogenized by an ultrasonic homogenizer and centrifuged at $4^{\circ} \mathrm{C}$. Solubilized FlicR1 protein in the supernatant was used to measure the fluorescence spectrum on a Safire2 plate reader (Tecan) and the absorbance spectrum on a DU-800 UVvisible spectrophotometer (Beckman).

Cell culture. HeLa cells (CCL-2; ATCC) were cultured in DMEM supplemented with 10\% FBS (Sigma-Aldrich), 2 mm GlutaMax (Invitrogen), and penicillin-streptomycin and the cells were incubated for $48 \mathrm{~h}$ at $37^{\circ} \mathrm{C}$ and $5 \% \mathrm{CO}_{2}$. Cells were split and cultured on collagen-coated 35 $\mathrm{mm}$ glass bottom dishes (Matsunami) to $\sim 50 \%$ confluency. Transfection was performed by incubating HeLa cells with the mixture of $1 \mu \mathrm{g}$ of plasmid DNA and $2 \mu \mathrm{l}$ of Turbofect (Thermo Fisher Scientific) for $2 \mathrm{~h}$ following the manufacturer's instructions. Imaging was performed 24-48 $\mathrm{h}$ after transfection.

Human embryonic kidney 293T (HEK293T) cells (CRL-11268; ATCC) were cultured and transfected following standard protocols (Kralj et al., 2012). Briefly, HEK293T cells were grown at $37^{\circ} \mathrm{C}$ and $5 \%$ $\mathrm{CO}_{2}$ in DMEM supplemented with $10 \%$ FBS and penicillin-streptomycin. Cells were transfected with 400 ng of DNA using Transit 293T (Mirus) following the manufacturer's instructions. After $24 \mathrm{~h}$, cells were replated onto glass-bottom dishes (D35-20-1.5-N; In Vitro Scientific) coated with Matrigel (BD Biosciences) at $\sim 10,000 \mathrm{cells} / \mathrm{cm}^{2}$. Measurements were performed $48 \mathrm{~h}$ after transfection.

Rat hippocampal neurons. Rat glial monolayers were prepared similarly to McCarthy and de Vellis (1980). Briefly, dissociated hippocampal cells from postnatal day $0(\mathrm{P} 0)$ rat pups of either sex (Sprague Dawley; Tocris Bioscience) (Banker and Goslin, 1998) were plated on a $10 \mathrm{~cm}$ culture dish in glial medium (GM) composed of 15\% FBS (Life Technologies), $0.4 \%$ (w/v) D-glucose, $1 \%$ Glutamax (Life Technologies), and 1\% penicillin-streptomycin (Life Technologies) in MEM (Life Technologies). After reaching confluence, cells were replated onto glass-bottomed dishes (D35-20-1.5-N; In Vitro Scientific) coated with Matrigel (BD Biosciences) at a density of 3500 cells $/ \mathrm{cm}^{2}$. When the glial monolayers reached confluence again, the medium was replaced by GM with $2 \mu \mathrm{M}$ cytarabine (cytosine- $\beta$-arabinofuranoside; Sigma-Aldrich). Dishes were maintained in GM with $2 \mu \mathrm{M}$ cytarabine until use.

Hippocampi dissected from P0 rat pups were dissociated using papain and plated in plating medium (PM) at $8000 \mathrm{cells} / \mathrm{cm}^{2}$ on the preestablished glial monolayers (Banker and Goslin, 1998). At $1 \mathrm{~d}$ in vitro (DIV), PM was exchanged for $2 \mu \mathrm{M}$ cytarabine in neurobasal-based medium (NBActiv4; Brainbits). Neurons were subsequently fed every $5 \mathrm{~d}$ by replacing $1 \mathrm{ml}$ of culture medium with NBActiv4 without cytarabine. Neurons were transfected on DIV 9 via calcium phosphate as described previously (Jiang and Chen, 2006). Measurements on neurons were performed on DIV 14. For all-optical electrophysiology experiments in neurons, cells were cotransfected by calcium phosphate with channelrhodopsin and FlicR1 DNA on DIV 9 and measured on DIV 12-13.

For measurements of spontaneous activity, dissociated E18 Sprague Dawley hippocampal cells in hibernate EB complete media were purchased from BrainBits. The cells were grown on $35 \mathrm{~mm}$ glass bottom dish (In Vitro Scientific) coated with poly-D-lysine (A-003-E; Millipore) containing $2 \mathrm{ml}$ of NbActiv4 (BrainBits) supplemented with 2\% FBS, penicillin-G potassium salt ( 25 units $/ \mathrm{ml})$, and streptomycin sulfate $(25$ $\mu \mathrm{g} / \mathrm{ml})$. Half of the culture medium was replaced every $3 \mathrm{~d}$. Neuronal cells were transfected on day 8 using Lipofectamine 2000 (Life Technologies) following the manufacturer's instructions. Briefly, $1-2 \mu \mathrm{g}$ of plasmid DNA and $4 \mu \mathrm{l}$ of Lipofectamine 2000 (Life Technologies) were added to $100 \mu \mathrm{l}$ of NbActive 4 medium to make the transfection medium. This medium was then incubated at room temperature for 10-15 min. Half of the culture medium $(1 \mathrm{ml})$ from each neuron dish was taken out and combined with an equal volume of fresh NbActiv4 medium (supplemented with $2 \%$ FBS, penicillin-G potassium salt, and streptomycin sulfate) to make a $1: 1$ mixture and incubated at $37^{\circ} \mathrm{C}$ and $5 \% \mathrm{CO}_{2}$. Next, 1 $\mathrm{ml}$ of fresh conditioned (at $37^{\circ} \mathrm{C}$ and $5 \% \mathrm{CO}_{2}$ ) NbActiv4 medium was then added to each neuron dish. The transfection medium was then added and the neuron dishes were incubated for $2-3 \mathrm{~h}$ at $37^{\circ} \mathrm{C}$ in a $\mathrm{CO}_{2}$ incubator. The medium was then replaced using the conditioned 1:1 medium prepared previously. The cells were incubated for $48-72 \mathrm{~h}$ at $37^{\circ} \mathrm{C}$ in a $\mathrm{CO}_{2}$ incubator before imaging.

Induced transmembrane voltage screening. For induced transmembrane voltage (ITV) screening, FlicR variants were coexpressed in HeLa cells, together with the inward rectifier potassium channel, Kir2.1 (Addgene \#32641) and ArcLight Q239 (Addgene \#36856) as an internal reference. Expression of Kir2.1 in HeLa cells helped maintain the resting potential of $\sim-60 \mathrm{mV}$, which is appropriate for screening neuronal voltage indicators. A uniform electric field of $\sim 50 \mathrm{~V} / \mathrm{cm}$ was applied across the cell culture to create an ITV. A pulse generator (PG 58A; Gould Advanced) was used to apply a $10 \mathrm{~ms}$ square-wave pulse at $\sim 0.5 \mathrm{~Hz}$. An amplifier (6824A 40V/25A; Agilent Technologies) was used to obtain a pulse amplitude of $25 \mathrm{~V}$. A pair of parallel platinum electrodes $(0.5 \mathrm{~cm}$ apart) were used to deliver the pulses to the cell culture. Fluorescence was imaged simultaneously with multiple electric field pulses for ITV at a frame rate of a $100 \mathrm{~Hz}$ for $10 \mathrm{~s}$.

Imaging ITV in HeLa cells. Imaging was performed in HEPES (25 mM)-buffered Hanks' balanced salt solution (HBSS). An inverted fluorescence microscope (Eclipse Ti-E; Nikon) equipped with a $200 \mathrm{~W}$ metal halide lamp (PRIOR Lumen) and a $60 \times$ oil objective was used to image $\mathrm{HeLa}$ cells. Images were acquired at $100 \mathrm{~Hz}$ with $4 \times 4$ binning using a 16-bit QuantEM 512SC electron-multiplying CCD camera (Photometrics). A FITC/Cy2 filter set (470/40 nm (excitation), 525/50 nm (emission), and a 495LP dichroic mirror (set number 49002; Chroma) was used to image ArcLight Q239. A TRITC/Cy3 filter set (545/30 nm (excitation), 620/60 nm (emission), and a 570LP dichroic mirror (set number 49005; Chroma) was used to image FlicR1. NIS Elements Advanced Research software (Nikon) was used to control the microscope and camera. The raw fluorescence traces of both FlicR and ArcLight were extracted from identical regions of interest in cells expressing both constructs and exported into a customized Microsoft Excel spreadsheet. Background subtraction, photobleaching corrections, calculations of average $\Delta F /$ $F_{\text {min }}$, and calculation of signal-to-noise ratios (SNRs) were performed automatically in Excel. The average $\Delta F / F_{\min }$ and SNR of FlicR signals were compared with those of ArcLight signals from the same cells and the ratios of $\Delta F / F_{\min }$ of FlicR versus ArcLight were reported. At least 10 cells coexpressing FlicR and ArcLight were analyzed for each variant. The best variant with maximum mean ratio in each library was determined and sequenced.

Imaging spontaneous activity in primary neuron culture. Imaging was performed in HEPES (25 mM)-buffered HBSS. Wide-field imaging was 
performed on an inverted Nikon Eclipse Ti-E microscope equipped with a $200 \mathrm{~W}$ metal halide lamp (PRIOR Lumen), 60× oil objectives (numerical aperture, $\mathrm{NA}=1.4$; Nikon), and a 16-bit QuantEM 512SC electronmultiplying CCD camera (Photometrics). A TRITC/Cy3 filter set (545/30 nm (excitation), 620/60 nm (emission), and a 570LP dichroic mirror (\#49005; Chroma) was used to image FlicR1. For time-lapse imaging, neurons were imaged at $100 \mathrm{~Hz}$ imaging frequency with $4 \times 4$ binning. Raw fluorescence traces were corrected for background autofluorescence and photobleaching. As seen in Figure 5A, FlicR1 shows some intracellular puncta. Mounting evidence points to these structures being lysosomes in which the protein is accumulating faster than it is being degraded (Katayama et al., 2008).

Simultaneous electrophysiology and fluorescence in HEK cells and primary neuron culture. All imaging and electrophysiology measurements were performed in Tyrode's buffer containing the following (in mM): 125 $\mathrm{NaCl}, 2.5 \mathrm{KCl}, 3 \mathrm{CaCl}_{2}, 1 \mathrm{MgCl}_{2}, 15$ HEPES, and 30 glucose, $\mathrm{pH}$ 7.3. For HEK cell measurements, 2-aminoethoxydiphenyl borate (100 $\mu \mathrm{M}$; Sigma-Aldrich) was added to block endogenous gap junctions. Measurements at $34^{\circ} \mathrm{C}$ were performed by perfusing in Tyrode's buffer at $1 \mathrm{ml} /$ min while maintaining elevated temperature with an inline heater (Warner Instruments) and an objective heater (Bioptechs).

Filamented glass micropipettes (WPI) were pulled to a tip resistance of 4-8 $\mathrm{M} \Omega$ and filled with internal solution containing the following (in $\mathrm{mm}$ ): 125 potassium gluconate, $8 \mathrm{NaCl}, 0.6 \mathrm{MgCl}_{2}, 0.1 \mathrm{CaCl}_{2}, 1 \mathrm{EGTA}, 10$ HEPES, $4 \mathrm{Mg}$-ATP, and 0.4 Na-GTP, pH 7.3, adjusted to $295 \mathrm{mOsm}$ with sucrose. Pipettes were positioned with a Sutter Instruments MP285 manipulator. Whole-cell, voltage and current-clamp recordings were acquired using an Axopatch 700B amplifier (Molecular Devices), filtered at $2 \mathrm{kHz}$ with the internal Bessel filter, and digitized with a National Instruments PCIE-6323 acquisition board at $10 \mathrm{kHz}$. Data were only acquired from cells with access resistance $<25 \mathrm{M} \Omega$. To determine the minimal current injection necessary for action potential generation before imaging, square waves of increasing amplitudes were injected in step sizes of $50-100 \mathrm{pA}$ while recording the voltage. The minimal current value that resulted in robust AP generation was used to generate action potentials for imaging experiments.

Illumination light was provided by a $561 \mathrm{~nm} 100 \mathrm{~mW}$ diode-pumped solid-state laser (Cobolt Jive $100561 \mathrm{~nm}$ ) for FlicR1 imaging and by a 488 $\mathrm{nm} 50 \mathrm{~mW}$ laser (Omicron PhoxX) for ArcLight imaging. The beam was expanded and focused onto the back-focal plane of a $60 \times$ oil-immersion objective (Olympus APON 60XOTIRF 1.49 NA). The imaging intensity at the sample was $10 \mathrm{~W} / \mathrm{cm}^{2}$. For all-optical electrophysiology measurements in neurons, the $488 \mathrm{~nm}$ beam was expanded and modulated by a digital micromirror device (Texas Instruments DLP LightCrafter Evaluation Module) controlled using built-in software. FlicR1 fluorescence was separated from excitation light and filtered using the dichroic and emission filters from a Cy3-4040C-OMF-ZERO filter set (Semrock). ArcLight fluorescence was separated with a long pass dichroic (Semrock catalog \#FF495-Di03) and filtered with a 531/40 nm band-pass filter (Semrock catalog \#FF01-531/40-25). The sample was imaged onto an EMCCD camera (Andor iXon ${ }^{+}$DU-860) with $128 \times 128$ pixels. For measurements of FlicRl's speed, movies were acquired at a $2 \mathrm{kHz}$ frame rate with $2 \times 2$ binning. Sensitivity measurements were acquired at $10 \mathrm{~Hz}$ with no binning. Photobleaching traces were acquired at $2 \mathrm{~Hz}$ with no binning. Neuronal data were acquired at $1 \mathrm{kHz}$ with $2 \times 2$ binning or at $500 \mathrm{~Hz}$ with $1 \times 1$ binning.

Some of the screening of all-optical electrophysiology conditions was performed on another custom-built microscope equipped with a higherresolution camera. Illumination light was provided by a $561 \mathrm{~nm} 100 \mathrm{~mW}$ Cobolt Jive (catalog \#0561-04-01-0100-500), a 488 nm $100 \mathrm{~mW}$ Coherent Obis (catalog \#1226419), or a $405 \mathrm{~nm} 30 \mathrm{~mW}$ Dragon Laser (catalog \#11042443). Laser lines were combined with dichroic mirrors and intensities were modulated using acousto-optical tunable filters (Gooch \& Housego, catalog \#TF525-250-6-3-GH18A or \#48058-2.5-.55-5W). The $488 \mathrm{~nm}$ laser line was expanded to illuminate the chip of a digital micromirror device (Texas Instruments DLP LightCrafter with DLP 0.3 WVGA chipset) that was subsequently reimaged onto the sample plane. The 561 and $488 \mathrm{~nm}$ lines were focused at the back focal plane of an APON 60XOTIRF 1.49 NA objective (Olympus). Collimated $405 \mathrm{~nm}$ laser light at the back focal plane of the objective was defocused to obtain a $5 \mu \mathrm{m}$ spot at the sample and was guided at the sample plane using galvonometric mirrors (Thorlabs catalog \#GVS202) located in a conjugate plane. Fluorescence light was separated from illumination light using a quadband dichroic mirror (Semrock catalog \#Di01-R405/488/561/ 635). The fluorescence light was passed through a knife-edge split into two channels using a dichroic mirror, which were recombined and reimaged onto two halves of the chip of a scientific CMOS camera (Hamamatsu ORCA-Flash 4.0). The splitting and recombining were accomplished using dichroic mirrors (Semrock FF662-FDi01). Green and orange fluorescence was filtered using a HQ550/50m band-pass filter (Chroma) for $488 \mathrm{~nm}$ excitation, or an ET595/50m bandpass filter (Chroma) for $561 \mathrm{~nm}$ illumination. $458 \mathrm{~nm}$ illumination light was provided by an LED (LED Supply 07040-PR000-B) positioned above the sample filtered by a D480/60x excitation filter (Chroma) and controlled by a four-channel LED driver (Thorlabs DC4104). Electrophysiological recordings on this microscope were performed as above, but using an Axopatch 200B amplifier and CV203BU headstage (Molecular Devices). Signals were filtered at $5 \mathrm{kHz}$ with the internal Bessel filter and digitized at $10 \mathrm{kHz}$ using a National Instruments PCIe-6259 acquisition board.

Measurements on ArcLightQ239 kinetics were performed using 488 $\mathrm{nm}$ illumination light from a Coherent Obis 488-50 laser focused onto the back focal plane of a $60 \times$ water-immersion objective (zcomOlympus UIS2 UPlanSApo $\times 60 / 1.20 \mathrm{~W}$, NA 1.20 ) to a power fluence of 2-4 $\mathrm{W} / \mathrm{cm}^{2}$ at the sample. Fluorescence light was split from the excitation light using a Semrock Di01-R405/488/594 dichroic, passed through a 525/30 band-pass emission filter, and imaged onto a scientific CMOS camera (Hamamatsu ORCA-Flash 4.0). Whole-cell voltage recordings were performed with a patch-clamp amplifier (A-M Systems Model 2400 ), filtered at $5 \mathrm{kHz}$ with the internal filter, and digitized with a National Instruments PCIE-6323 acquisition board at $10 \mathrm{kHz}$.

FlicR's two-photon voltage sensitivity was tested on a homebuilt beam-scanning two-photon microscope with an $80 \mathrm{MHz}, 100$ fs tunable pulsed laser (SpectraPhysics Insight DeepSee). Measurements were performed at $1120 \mathrm{~nm}$ excitation wavelength with a time-averaged excitation power of $60 \mathrm{~mW}$, or $0.8 \mathrm{~nJ}$ per pulse, in imaging mode, and $\sim 6 \mathrm{~mW}$ or $80 \mathrm{pJ}$ per pulse in point-recording mode. Pulses were focused down to an $\sim 500 \mathrm{~nm}$ spot with a 1.2 NA water-immersion objective (Olympus UplanSapo). Imaging measurements were performed with linear scan speeds of $\sim 8 \mathrm{~cm} \mathrm{~s}^{-1}$. Excitation light and fluorescence were separated using an FF775-Di01 dichroic mirror and FF01-790/SP-25 short-pass filter (both Semrock). Fluorescence was detected using a Hamamatsu R943-02 photomultiplier tube in photon counting mode cooled to $-20^{\circ} \mathrm{C}$. The PMT signal was amplified through an SRS PR325 amplifier and discretized with a Hamamatsu Photon counting unit C9744. Data were acquired using an NI pci-6259 DAQ card. The setup was controlled by Labview software written in house.

HeLa cell all optical electrophysiology. Imaging was performed in HEPES (25 mM)-buffered HBSS. An inverted fluorescence microscope (Eclipse Ti-E; Nikon) equipped with a $200 \mathrm{~W}$ metal halide lamp (PRIOR Lumen) and a $60 \times$ oil objective was used. Images were acquired at 100 $\mathrm{Hz}$ with $4 \times 4$ binning using a 16-bit QuantEM 512SC electronmultiplying CCD camera (Photometrics). Cells expressing R-GECO1 only, R-GECO1 and ChIEF-Citrine, FlicR1 only, or FlicR1 and ChIEFCitrine were exposed to whole-field illumination with pulses of blue light ( $405 \mathrm{~nm}$ laser, $20 \mathrm{~ms}, 5 \mathrm{~Hz}$ and $10 \mathrm{~Hz}, 5 \mathrm{~mW} / \mathrm{mm}^{2}$ ) to stimulate ChIEF. Cells were simultaneously illuminated with yellow light to excite fluorescence of R-GECO1 or FlicR1. A TRITC/Cy3 filter set (545/30 nm (excitation), 620/60 nm (emission), and a 570LP dichroic mirror (\#49005; Chroma) was used to image R-GECO1 or FlicR1 fluorescence. The NIS Elements Advanced Research software (Nikon) was used to control the microscope and camera. Fluorescence changes were calculated by averaging over the whole cell. Raw fluorescence traces were corrected for background autofluorescence and photobleaching.

Different opsins were explored in an effort to minimize optical crosstalk with FlicR1. The yellow light intensity used to image FlicR1 in HeLa cells at $100 \mathrm{~Hz}$ acquisition rate $\left(60 \mathrm{~mW} / \mathrm{cm}^{2}\right)$ is much lower than that required to image AP transients in neurons $\left(10 \mathrm{~W} / \mathrm{cm}^{2}\right)$. As mentioned above, we used ChIEF in our proof-of-concept experiments because 60 
$\mathrm{mW} / \mathrm{cm}^{2}$ of yellow light is not enough to cause activation of ChIEF. However, we had to switch to more blue-shifted opsins (TsChR and PsChR) to try to avoid optical crosstalk with the relatively higher illumination intensity required to image FlicR 1 in neurons at $1 \mathrm{kHz}$ (see Fig. 8).

Preparation of rat hippocampal organotypic brain slices. Horizontal brain slices (250 $\mu \mathrm{m}$ thickness) from a P0 Sprague-Dawley rat of either sex were generated in ice-cold HBSS containing $1.3 \mathrm{mM} \mathrm{CaCl}_{2}$ and $1 \mathrm{~mm}$ $\mathrm{MgSO}_{4}$ with a vibrating microtome (Leica VT1000S) as described previously (Panaitescu et al., 2013). All procedures were performed in compliance with the guidelines of the Canadian Council for Animal Care and with the approval of the University of Alberta Animal Care and Use Committee for Health Science. Hippocampal regions were cut from horizontal brain slices and placed on a sterile $0.4 \mu \mathrm{m}$ pore membrane cell culture insert (Millipore PICMORG50). The insert and slice were then placed in a Petri dish containing $1.5 \mathrm{ml}$ of NbActiv4 (BrainBits) supplemented with $5 \%$ FBS, penicillin-G potassium salt (50 units $/ \mathrm{ml}$ ), and streptomycin sulfate $(50 \mu \mathrm{g} / \mathrm{ml})$. Slices were cultured at $37^{\circ} \mathrm{C}$ and $5 \%$ $\mathrm{CO}_{2}$ for $3-5 \mathrm{~d}$ before transfection.

Ex vivo electroporation of organotypic brain slices. The Millipore insert and the slice cultured on it were placed between a platinum plate Petri dish electrode (CUY700-P2E; Nepa Gene) and a square platinum electrode (CUY700-P2L; Nepa Gene). The gap between the dish electrode and the membrane was filled with electroporation buffer (HBSS with 1.5 $\mathrm{mm} \mathrm{MgCl}_{2}$ and $10 \mathrm{~mm}$ glucose). Plasmid pAAV2-hSyn-FlicR1 was dissolved in electroporation buffer at a concentration of $1 \mu \mathrm{g} / \mu \mathrm{l}$ and sufficient volume was added to fill the gap between the slice and the top electrode. Five $20 \mathrm{~V}$ pulses $(5 \mathrm{~ms}$ each, $1 \mathrm{~Hz}$ ) were applied using a function generator (PG 58A; Gould) and an amplifier (6824A; Agilent Technologies). The direction of electrical field was then reversed and a second set of five pulses with the same settings were applied. After transfection, the slices were returned to the incubator at $37^{\circ} \mathrm{C}$ with $5 \% \mathrm{CO}_{2}$. It normally takes $2-3 \mathrm{~d}$ for full expression of FlicR1 in cultured brain slices using this transfection method.

Imaging of rat organotypic hippocampal slices. For organotypic brain slice imaging, an upright FV1000 confocal microscope (Olympus) equipped with FluoView1000 software (Olympus) and a 20× XLUMPlanF1 waterimmersion objective (NA 1.00; Olympus) was used. Illumination light was provided by a $100 \mathrm{~W}$ mercury arc lamp (Olympus).

The brain slice on the Millipore insert was placed in a custom-made chamber to hold it in place during imaging. Immediately before imaging, the slices were perfused with artificial CSF (ACSF) superfusate containing the following (in mM): $120 \mathrm{NaCl}, 3 \mathrm{KCl}, 1 \mathrm{CaCl}_{2}, 2 \mathrm{MgSO}_{4}, 26 \mathrm{NaHCO}_{3}, 1.25$ $\mathrm{NaH}_{2} \mathrm{PO}_{4}$, and 10 D-glucose, $\mathrm{pH}$ adjusted to 7.4 by gassing with $95 \% \mathrm{O}_{2}, 5 \%$ $\mathrm{CO}_{2}$, at $5 \mathrm{ml} / \mathrm{min}$ using a peristaltic pump (Watson-Marlow Alitea) and buffer temperature was controlled at $34^{\circ} \mathrm{C}$. Imaging was started within 10 min after activation of the perfusion system.

To image FlicR1, the hippocampal slice was excited with the $100 \mathrm{~W}$ mercury arc lamp using a filter cube with $565 / 30 \mathrm{~nm}$ excitation filter, a $620 / 50 \mathrm{~nm}$ emission filter and $585 \mathrm{~nm}$ dichroic (Semrock). Images were acquired at $100 \mathrm{~Hz}$ at $2 \times 2$ binning (see Fig. $6 \mathrm{~B}$, spontaneous activity) and $50 \mathrm{~Hz}$ (see Fig. 6E, stimulated activity) using a digital sCMOS camera (Hamamatsu Orca-Flash2.8; Hamamatsu Photonics). For stimulation with theophylline, $\sim 30 \mathrm{~s}$ after the start of the experiment, the superfusate was changed from control ACSF to ACSF containing $10 \mathrm{~mm}$ theophylline (Sigma-Aldrich, directly dissolved in ACSF). Approximately $10 \mathrm{~min}$ later, the superfusate was changed back to control ACSF. Four organotypic brain slices transfected with FlicR1 were imaged and showed similar responses. Figure 6 shows representative fluorescence traces from neurons in organotypic slices.

Data analysis. For measurements of speed and sensitivity in HEK cells, fluorescence traces were extracted using the maximum likelihood pixel weighting algorithm described previously (Kralj et al., 2012). Briefly, each pixel's fluorescence was correlated with the average whole-field fluorescence. Pixels with stronger correlation to the mean were weighted preferentially in the measurement of fluorescence, emphasizing the pixels containing the most information. The $5 \%$ of pixels with the highest correlation to the mean were used for characterization of protein speed and sensitivity. Fluorescent responses to step functions were averaged over 100 trials and fit with a double exponential function to acquire the time constants of FlicR1's fluorescent response. Sensitivities were defined as the maximum percentage change in fluorescence over a $150 \mathrm{mV}$ change in voltage.

Photobleaching traces were obtained by subtracting the average fluorescence of a region of user-defined background region from the average fluorescence of the user-defined region of the cell. The resulting traces were then fit to a single exponential with a baseline offset to obtain the photobleaching time constant. FlicR1 showed transient photoactivation in the first $100 \mathrm{~s}$, which was ignored for photobleach calculations.

For neuronal measurements, the cell body and dendrites were selected manually and the average intensity of all included pixels was averaged. The background intensity of a user-defined background region was subtracted from the raw signal. A photobleaching baseline was constructed from the whole-field intensity by a sliding minimum filter, followed by a sliding mean filter. Each frame of the movie was then corrected by dividing by this baseline. SNRs were calculated as the maximum fluorescence response to action potential (as identified by the patch-clamp recording) divided by the SD of the baseline fluorescence.

Data were analyzed using custom MATLAB codes and Microsoft Excel. A Q-Q plot was used to test the normality of datasets being compared. Both datasets were determined to have equal variance using the $F$ test $(\alpha=0.05)$. Unless noted otherwise, values reported are mean \pm SEM. A statistical method was not used to justify sample sizes, but sample sizes are similar to those used by others in the field. Data selection criteria for experiments are stated in the Materials and Methods section for each experiment and are similar to those used by others in the field.

\section{Results \\ Development of FlicR}

The first step for creating the voltage indicator was to make a chimera from the genes for a red-shifted FP and a voltagesensitive domain (Fig. 1A,B). Our choice of the red-shifted FP to use as the template was inspired by the $\mathrm{x}$-ray crystal structure (Akerboom et al., 2013) of the $\mathrm{Ca}^{2+}$ indicator R-GECO1 (Zhao et al., 2011). The structure reveals that the Lys80 (R-GECO1 numbering) side chain from the surface of the cpmApple-derived $\beta$-barrel engages in an electrostatic interaction with the phenolate oxygen of the chromophore. This structural evidence, combined with additional mutagenesis results (Wu et al., 2014), points to this interaction as the mechanistic basis for the response of R-GECO1. Because the lysine is part of the $\beta$-barrel (in contrast to GCaMP, in which the key interaction is with an arginine from CaM; Wang et al., 2008), we reasoned that the cpmApple domain is likely a self-contained unit that would retain its fluorescence modulation mechanism even if the $\mathrm{Ca}^{2+}$ sensitive domain were replaced with an alternative sensing domain.

We chose to use CiVSD as the voltage-sensitive domain to fuse to cpmApple because it localizes properly to the cell membrane of mammalian cells, as demonstrated by its successful use in recent FP-based voltage indicators (Lundby et al., 2008; Gautam et al., 2009; Akemann et al., 2012; Barnett et al., 2012; Jin et al., 2012; Lam et al., 2012). We elected to use a cpFP to increase the chance of coupling voltage-induced conformational changes in the VSD to a change in the FP chromophore environment, likely through its influence on the conformation of the Lys80 side chain. Specifically, fusion of cpmApple to the $\mathrm{C}$ terminus of CiVSD should bring Lys80 and the chromophore in close proximity to the mobile S4 helix of the VSD domain. We hypothesized that this design should maximize the likelihood of developing an indicator with fast kinetics because movement of S4 could influence the chromophore environment directly.

Although directed protein evolution has been one of the main strategies to improve FP properties and FP-based $\mathrm{Ca}^{2+}$ indicators (Zhao et al., 2011; Chen et al., 2013), it has not been widely implemented for voltage indicators. To facilitate our directed 
A

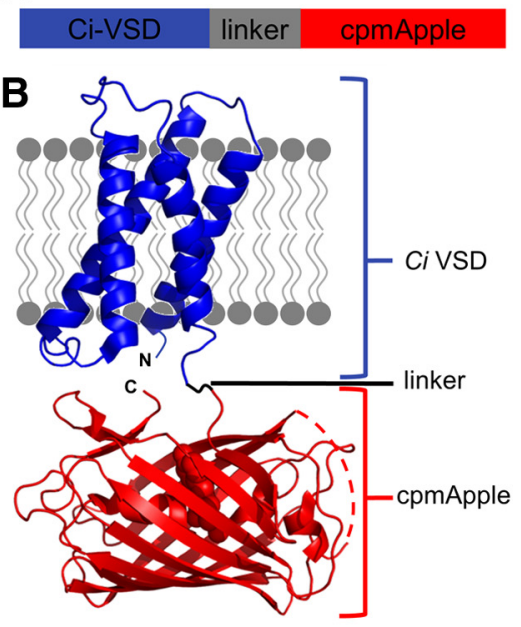

C

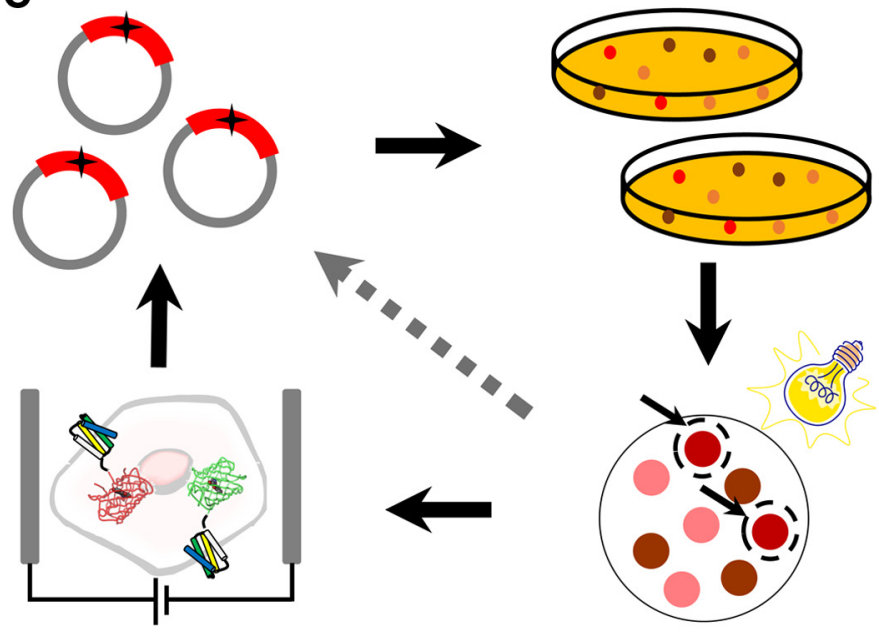

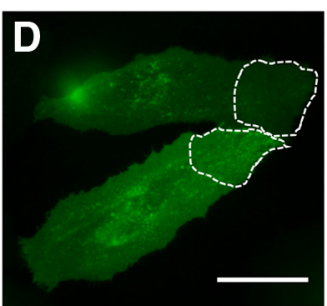

$\mathbf{F}$
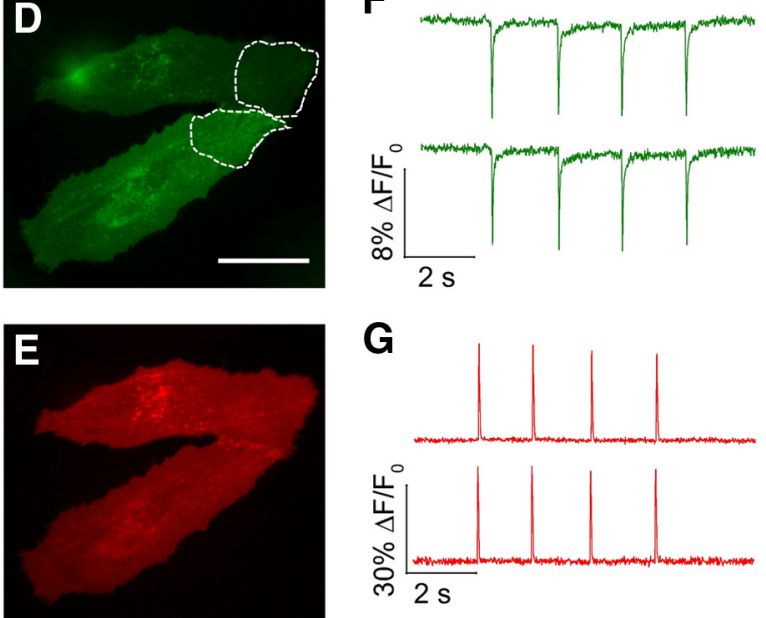

G

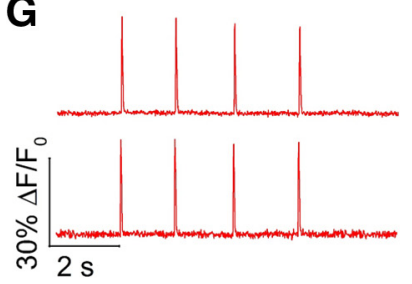

H



Figure 1. Schematic representation of FlicR indicator and directed evolution process. $A, B$, Representation of FlicR indicator. $\boldsymbol{B}$, Model of FlicR1 represented by the crystal structures of CiVSD (PDB ID 4G80; Li et al., 2014) and cpmApple (PDB ID 4I2Y; Akerboom et al., 2013). C, Schematic representation of directed evolution strategy used to develop FlicR1. First, libraries of DNA-encoding indicator genes were transformed into E. coli and cultured on agar plates. Second, E. coli colonies expressing FlicR1 were illuminated with yellow light. Colonies with bright red fluorescence were picked and screened for voltage sensitivity in mammalian cells. Voltage sensitivity of FlicR1 variants was then tested via field stimulation in HeLa cells coexpressing ArcLight. $\boldsymbol{D}, \boldsymbol{E}$, Image of HeLa cells coexpressing ArcLight Q239 (D) and FlicR1 (E), Scale bar, $10 \mu \mathrm{m}$. F, ArcLight fluorescence response of two regions shown in D to electrical field stimulation pulses (10 ms, $25 \mathrm{~V})$. G, FlicR1 fluorescence response of the same two regions shown in $\boldsymbol{D}$ to electrical field stimulation pulses $(10 \mathrm{~ms}, 25 \mathrm{~V})$. $\boldsymbol{H}$, Improvement in voltage sensitivity of FlicR variants during directed evolution represented as a ratio of response amplitude in HeLa cells compared with ArcLight Q239. Error bars indicate SD ( $n=10-15$ cells). Fluorescence imaging for field stimulation measurements was performed at $100 \mathrm{~Hz}$. Illumination intensities were $0.2 \mathrm{~W} / \mathrm{cm}^{2}$ for FlicR1 and $0.1 \mathrm{~W} / \mathrm{cm}^{2}$ for Arclight Q239.

evolution strategy, we designed a high-throughput workflow for screening of voltage indicators (Fig. 1C). This procedure was divided into two steps: an E. coli bacterial colony screen for brightness and a mammalian cell screen for voltage sensitivity. The second step of the screen also enabled us to assess the membrane localization of the engineered variants so that variants that do not localize properly can be excluded from further rounds of screening. In brief, libraries of genes encoding voltage indicator variants were inserted in a dual bacterial and mammalian expression vector and used to transform E. coli. After overnight growth on agar plates, the red fluorescence of the colonies was imaged using a custom fluorescence imaging setup (Cheng and Campbell, 2006) that can image $\sim 1000$ colonies simultaneously, enabling the screening of tens of thousands of variants per round. The brightest variants were cultured and their plasmid DNA was used to transfect HeLa cells coexpressing ArcLight Q239 and an inward-rectifying potassium channel (Kir2.1). ArcLight Q239 provides an internal reference to assess voltage sensitivity. Kir2.1 hyperpolarizes the resting membrane potential of HeLa cells to -60 mV (Kirkton and Bursac, 2011; Hochbaum et al., 2014; Zou et al., 2014) to mimic that of neurons. The transfected cells were subjected to $10 \mathrm{~ms}$ electrical field pulses using a custombuilt field stimulation system and fluorescence response to stimulation was recorded (Fig. $1 D-G$ ). FlicR variants with the highest voltage sensitivity were selected to go on to the next round of directed evolution.

For our first-generation construct, we fused cpmApple (residues 60-304, R-GECO1 numbering (Akerboom et al., 2013; Fig. 2) to the $C$ terminus of CiVSD truncated at residues 236-242 to construct seven variants. We chose these positions to bring cpmApple as close as possible to the S4 helix. The shortest linker that exhibited fluorescence was the variant with CiVSD truncated at 239. Our experience with evolving FP-based indicators (Zhao et al., 2011; Jin et al., 2012; Han et al., 2013; Wu et al., 2013) has shown that even small adjustments in the linker between the sensing domain and the FP are critical to the indicator function. 


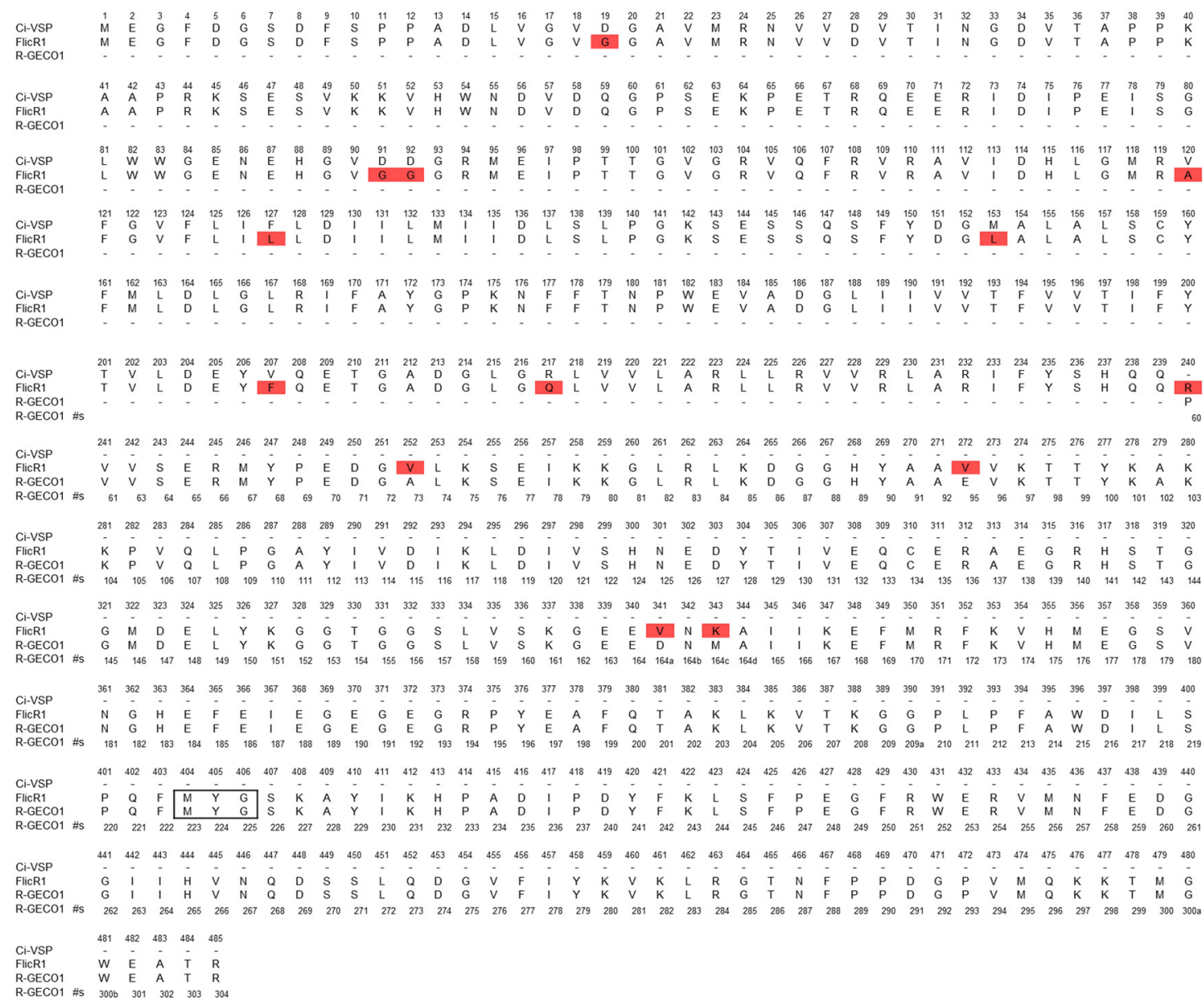

Figure 2. Sequence alignment of FlicR1. Alignment of FlicR1 gene with the CiVSD domain (top) and cpmApple (residues 60-304, R-GEC01 numbering) from R-GEC01 (bottom). The red highlighted residues are the amino acid mutations of FlicR1 compared with the starting template. Residues MYG in the box correspond to the mApple chromophore. (almodulin (CaM) and the M13 peptide are not shown in the R-GECO1 sequence.

In an effort to identify the optimal composition of the linker between CiVSD and the cpmApple domain, we created a library of 1024 gene variants ( 400 protein variants) by fully randomizing the codons for two residues immediately after residue 239 of CiVSD (Pro240 and Val241, respectively). Screening of this library for red fluorescence, followed by voltage sensitivity tests in HeLa cells subjected to field stimulation, led to the identification of a variant with mutation Pro240Arg. We designated this variant as our first-generation FlicR. This FlicR variant (FlicR0.1) exhibited $\operatorname{dim}$ fluorescence that was responsive to membrane potential changes in mammalian cells, albeit with a small response amplitude (Fig. 1H). Notably, FlicR0.1 (and all subsequent variants) exhibits an increase in fluorescence in response to membrane depolarization, whereas ArcLight and ASAP1 exhibit decreases in fluorescence. To evolve FlicR0.1 brightness and voltage sensitivity, we used both rational design and directed protein evolution by multiple rounds of construction and screening of libraries containing thousands of variants.

In a typical round of directed evolution, we used error-prone PCR to create a large library based on our most promising template(s) and then screened the library using the workflow described above. A mixture of the three to six variants with the brightest fluorescence, proper membrane localization, and largest responses to voltage change would then be used as the template for the next round of library creation by random mutagenesis. Four rounds of this procedure led to FlicR0.5, which was $16 \pm 3$-fold brighter than FlicR0.1
( $n=100$ colonies $)$ and could easily be imaged with wide-field fluorescence microscopy when expressed in mammalian cells. Three more rounds of evolution were then performed, with greater emphasis placed on identifying variants with improved voltage sensitivity rather than improved brightness. This effort led to the identification of the Val207Ala mutation, located in the S3 to S4 linker of the CiVSD, which provided a pronounced improvement in voltage sensitivity. For the eighth round of evolution, we mutated position 207 to all 20 possible amino acids and screened the library as before. The best variant identified from this library harbored the Val207Phe mutation that further improved the voltage sensitivity (Fig. $1 E, G$ ). This variant, which has 12 mutations relative to FlicR0.1 (Fig. 2), was designated as FlicR1. Relative to ArcLight Q239, FlicR1 has a $3.6 \pm 0.4$-fold ( $n=12 \mathrm{HeLa}$ cells) greater fluorescence response to a $10 \mathrm{~ms}$ electrical pulse (Fig. $1 D-H)$. When stimulated with a train of electrical pulses at $10 \mathrm{~Hz}$, FlicR 1 fluorescence response showed faster response and decay kinetics compared with ArcLight Q239, which did not return to baseline between stimulations.

\section{Characterization of FlicR1 in HEK cells}

To quantify the voltage sensitivity and speed of FlicR1, we expressed it under a CMV promoter in HEK cells and used wholecell voltage clamp to change membrane potential while recording FlicR1 fluorescence under $561 \mathrm{~nm}$ illumination $\left(10 \mathrm{~W} / \mathrm{cm}^{2}\right)$. FlicR1 trafficked well to the membrane of HEK cells (Fig. $3 A$ ). When we applied a triangle wave of membrane voltage from 




B
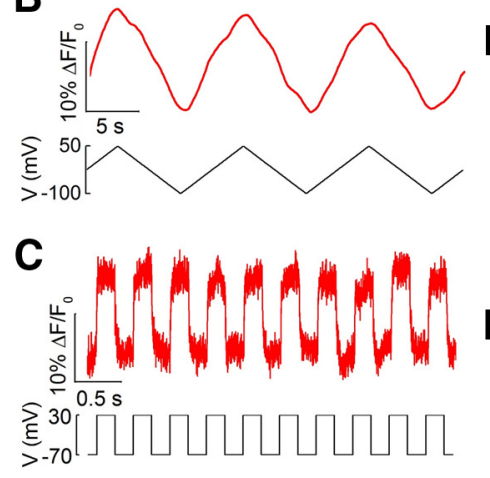

I

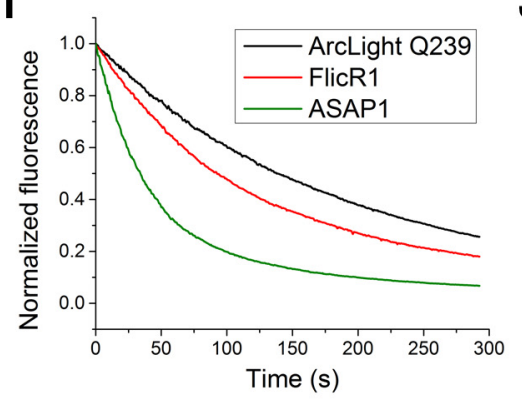

D

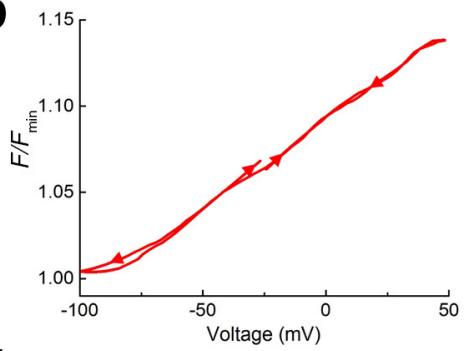

F

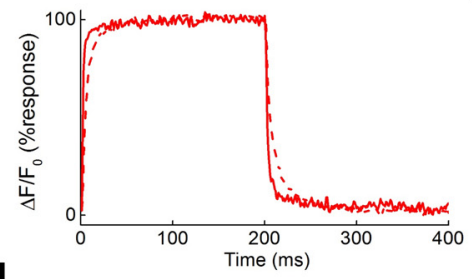

H

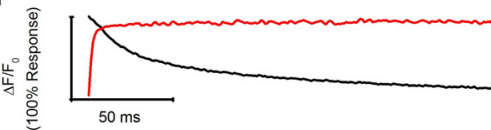

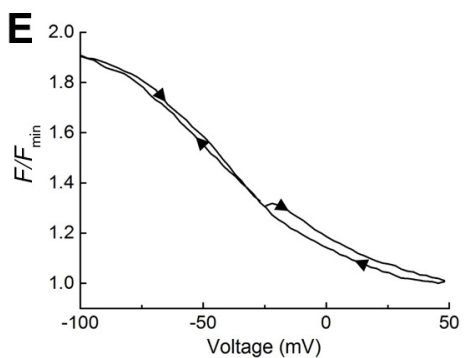

G
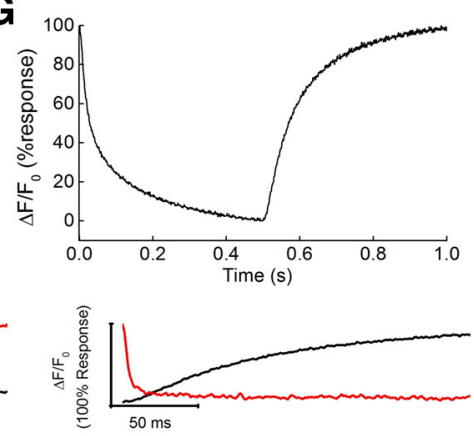

K

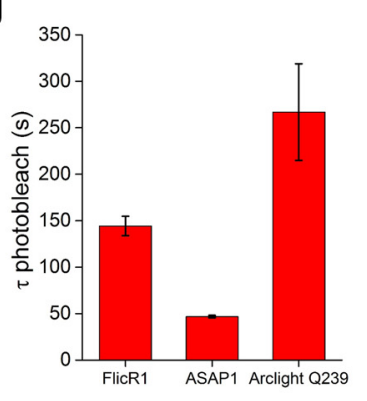

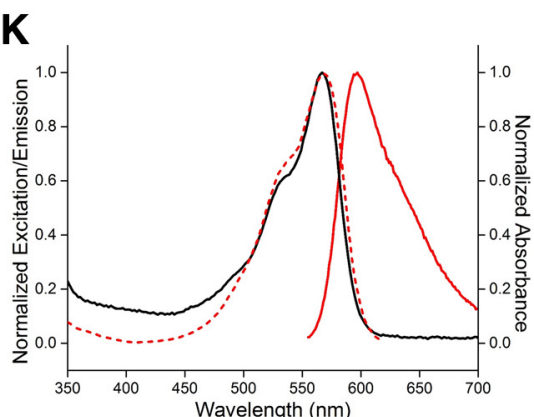

Figure 3. Characterization of FlicR1. $\boldsymbol{A}$, Image of HEK293 cells expressing FlicR1 under the CMV promoter. Scale bar, $10 \mu \mathrm{m}$. $\boldsymbol{B}$, Fluorescence response (top) to a triangle wave in membrane potential (bottom) from $-100 \mathrm{mV}$ to $+50 \mathrm{mV}$. Fluorescence trace (acquired at $10 \mathrm{~Hz}$ ) is filtered using a 15 point moving-average low-pass filter. $\Delta F / F_{0}$ is calculated as $\left(F-F_{0}\right) / F_{0}$, where $F$ is the fluorescence at a particular time point and $F_{0}$ is initial fluorescence. C, FlicR1 fluorescence response (top) from a representative cell to a square wave in membrane potential (bottom) from $-70 \mathrm{mV}$ to $+30 \mathrm{mV}$. D, E, FlicR1 (D) and ArcLight Q239 (E) fluorescence as a function of membrane voltage in a representative HEK293 cell. Fluorescence $\left(F / F_{\text {min }}\right.$, where $F$ is the fluorescence intensity at a particular membrane voltage and $F_{\min }$ is the minimum fluorescence intensity over the range of $-100 \mathrm{mV}$ to $+50 \mathrm{mV}$ ) is the mean of three ramp cycles from $-100 \mathrm{mV}$ to $+50 \mathrm{mV}$ and back. Fluorescence is plotted starting at $-25 \mathrm{mV}$, depolarizing to $+50 \mathrm{mV}$, hyperpolarizing to $-100 \mathrm{mV}$, and then returning back up to $-25 \mathrm{mV}$ as marked by the arrows. Fluorescence showed little hysteresis between increasing and decreasing voltage ramps. $\boldsymbol{F}$, FlicR1 fluorescence response to a $100 \mathrm{mV}$ step potential in HEK 293 cells. Solid line shows fluorescence response at $34^{\circ} \mathrm{C}$. Dotted line shows fluorescence response at $22^{\circ} \mathrm{C}$. G, ArcLight $Q 239$ fluorescence response to a $100 \mathrm{mV}$ step potential in HEK293 cells $22^{\circ} \mathrm{C}$. Note the different time axis compared with $\boldsymbol{F}$. $\boldsymbol{H}$, Magnification of the "on" and "off" portions of $22^{\circ} \mathrm{C}$ fluorescence traces from FlicR (red) and ArcLight (black). I, Normalized bleaching curves for FlicR1, ArcLight, and ASAP1 in HEK239 cells. J, Time constants for photobleaching of FlicR1, ASAP1, and ArcLight Q239 in HEK293 cells using continuous $10 \mathrm{~W} / \mathrm{cm}^{2} 561 \mathrm{~nm}$ light illumination for FlicR1 and continuous $10 \mathrm{~W} / \mathrm{cm}^{2} 488 \mathrm{~nm}$ light illumination for ASAP1 and ArcLight Q239. Fluorescence was captured every $500 \mathrm{~ms}$. Time constants are based on single exponential fits. Error bars indicate SEM for FlicR1 ( $n=5$ cells), ASAP1 ( $n=5$ cells), and ArcLight Q239 ( $n=4$ cells). $\boldsymbol{K}$, Spectral characterization of FlicR1 in vitro. Shown are absorbance (solid black line), excitation (dotted red line), and emission (solid red line) of FlicR1. Fluorescence imaging for voltage-sensitivity measurements was performed at $10 \mathrm{~Hz}$. Step responses were recorded at $2 \mathrm{kHz}$ for FlicR1 and $1 \mathrm{kHz}$ for ArcLight Q239. Illumination intensities were $10 \mathrm{~W} / \mathrm{cm}^{2}$.

$-100 \mathrm{mV}$ to $+50 \mathrm{mV}$, fluorescence increased almost linearly with membrane voltage, with a fluorescence change $\left(\Delta F / F_{0}\right)$ of $6.6 \pm 0.6 \%(n=5)$ per $100 \mathrm{mV}$ (Fig. $3 B, D)$. This sensitivity is almost an order of magnitude larger than previously reported cpRFP-based voltage sensors (Gautam et al., 2009). To measure the kinetics of FlicR1, we stepped membrane voltage between -70 and $+30 \mathrm{mV}$ at $22^{\circ} \mathrm{C}$ (Fig. $3 \mathrm{C}$ ). These steps induced a rapid biexponential response in the fluorescence of FlicR1, with a dominant fast component that had a $3.0 \pm 0.2 \mathrm{~ms}$ time constant accounting for $90 \%$ of the activation response. For deactivation, the fast component made up $70 \%$ of the response and had a time constant of $2.8 \pm 0.3 \mathrm{~ms}$ (Fig. 3F, Table 1). These values compare favorably to ArcLight Q239 kinetics at $22^{\circ} \mathrm{C}$, which follow a biexponential response to the same protocol with time constants of $28 \pm 8 \mathrm{~ms}(39 \%)$ and $271 \pm 6 \mathrm{~ms}(61 \%)$ for the rising edge and of $100 \pm 20 \mathrm{~ms}(61 \%)$ and $280 \pm 40 \mathrm{~ms}(39 \%)(n=6)$ for deactivation (Fig. $3 E, G, H$, Table 1). We repeated the FlicR1 measure-
Table 1. FlicR1 and ArcLightQ239 kinetics in HEK293 cells

\begin{tabular}{|c|c|c|c|c|}
\hline & \multicolumn{2}{|l|}{$22^{\circ} \mathrm{C}$} & \multicolumn{2}{|l|}{$34^{\circ} \mathrm{C}$} \\
\hline & Activation & Deactivation & Activation & Deactivation \\
\hline & $\begin{array}{l}-70 \mathrm{mV} \\
\text { to } 30 \mathrm{mV}\end{array}$ & $\begin{array}{l}30 \mathrm{mV} \\
\text { to }-70 \mathrm{mV}\end{array}$ & $\begin{array}{l}-70 \mathrm{mV} \\
\text { to } 30 \mathrm{mV}\end{array}$ & $\begin{array}{l}30 \mathrm{mV} \\
\text { to }-70 \mathrm{mV}\end{array}$ \\
\hline \multicolumn{5}{|l|}{ FlicR1 } \\
\hline$\tau 1$ fast (ms) & $3.0 \pm 0.2$ & $2.8 \pm 0.3$ & $0.74 \pm 0.06$ & $0.93 \pm 0.07$ \\
\hline$\tau 2$ slow (ms) & $41 \pm 7$ & $18 \pm 3$ & $27 \pm 6$ & $14 \pm 6$ \\
\hline$\%$ fast component & $90 \pm 2$ & $70 \pm 4$ & $90 \pm 2$ & $79 \pm 4$ \\
\hline$n$ & \multicolumn{2}{|c|}{5} & \multicolumn{2}{|c|}{6} \\
\hline \multicolumn{5}{|l|}{ ArclightQ239 } \\
\hline$\tau 1$ (ms) & $28 \pm 8$ & $100 \pm 20$ & $9 \pm 1$ & $17 \pm 1$ \\
\hline$\tau 2$ (ms) & $271 \pm 6$ & $280 \pm 40$ & $48 \pm 4$ & $60 \pm 7$ \\
\hline$\%$ fast component & $39 \pm 8$ & $61 \pm 15$ & $50 \pm 3$ & $79 \pm 3$ \\
\hline$n$ & \multicolumn{2}{|c|}{6} & \multicolumn{2}{|c|}{6} \\
\hline
\end{tabular}

Values for Arclight Q239 at $34^{\circ} \mathrm{C}$ are from Jin et al. (2012). 
ments at $34^{\circ} \mathrm{C}$ and found that the time constant of the fast component decreased to $0.74 \pm 0.06 \mathrm{~ms}(90 \%)$ for activation and $0.93 \pm 0.07 \mathrm{~ms}(79 \%)$ for deactivation $(n=6)$ (Fig. $3 F$, Table 1$)$. The sensitivity at this temperature was $6 \pm 1 \%$ per $100 \mathrm{mV}$ and was not significantly different from the value at $22^{\circ} \mathrm{C}(n=6, p=0.49$, two-tailed Student's $t$ test).

We compared the rate of photobleaching of FlicR1 with other FP-based voltage indicators using $10 \mathrm{~W} / \mathrm{cm}^{2}$ of either 561 $\mathrm{nm}$ (FlicR1) or $488 \mathrm{~nm}$ light (ASAP1 and ArcLight Q239) (Fig. 3I). Single exponential fits gave photobleaching time constants of $150 \pm 10 \mathrm{~s}$ ( $n=5$ cells $)$ for FlicR1, $47 \pm 1 \mathrm{~s}(n=5$ cells $)$ for ASAP1, and $300 \pm 30 \mathrm{~s}(n=4$ cells $)$ for ArcLight Q239 (Fig. 3J). Measurement of the absorbance, excitation, and emission spectra of FlicR1 in vitro (Fig. $3 \mathrm{~K}$ ) revealed excitation and emission maxima of 570 and $597 \mathrm{~nm}$, respectively.

The fact that the absorption spectrum of FlicR1 is red shifted relative to other FP-based voltage sensors made it a promising candidate for two-photon excitation in the 1100-1300 $\mathrm{nm}$ range. To test the utility of FlicR1 for two-photon imaging, a two-photon scanning-beam microscope set to deliver $1120 \mathrm{~nm}$ excitation pulses was used to image HEK cells expressing FlicR1. Under whole-cell voltage clamp, the membrane voltage was varied in a square wave between -100 and $+100 \mathrm{mV}$ at $2 \mathrm{~Hz}$. FlicR 1 responded with a fluorescence change $\Delta F / F$ of $9 \pm 1 \%$ per $100 \mathrm{mV}$ $(n=8$ cells) (Fig. $4 A)$.

The speed of the FlicR1 response to a voltage step under two-photon excitation was tested in point-recording mode. Cells were subjected to a square wave in voltage from -100 to $+100 \mathrm{mV}$ at $100 \mathrm{~Hz}$ and fluorescence was recorded at $1 \mathrm{kHz}$ from a single point on the cell membrane (Fig. $4 B)$. FlicR1 is an order of magnitude more stable to two-photon photobleaching compared with ASAP1 (Fig. 4C), which enabled us to do point measurements. FlicR1 was fast and sensitive enough to resolve these voltage changes, although the signal amplitude was limited to $\sim 2 \%$ per $200 \mathrm{mV}$ at $100 \mathrm{~Hz}$ (Fig. 4B). Due to rapid photobleaching under static two-photon illumination, we did not pursue two-photon voltage imaging further. However, imaging with a beam that rapidly traces the contour of a cell membrane could ameliorate photobleaching and provide a viable option for further exploration (Brinks et al., 2015).

\section{Imaging and characterization of FlicR1 in cultured neurons}

An important benchmark for a voltage indicator is to resolve single action potentials in single neurons with single-trial recording. Based on the characterization in HEK cells, we reasoned that the combination of the high fluorescence response and fast kinetics of the FlicR1 fluorescence signal would allow it to record neuronal activity in single-trial recordings. To test this hypothe$\sim 400$ counts per bin initially.
B
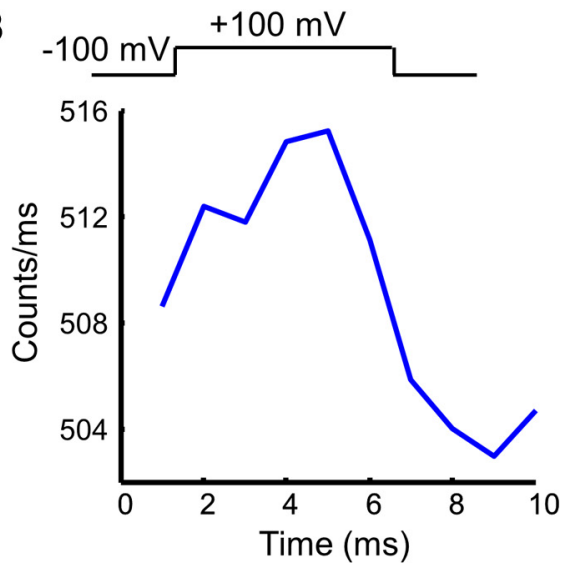

Time (s)

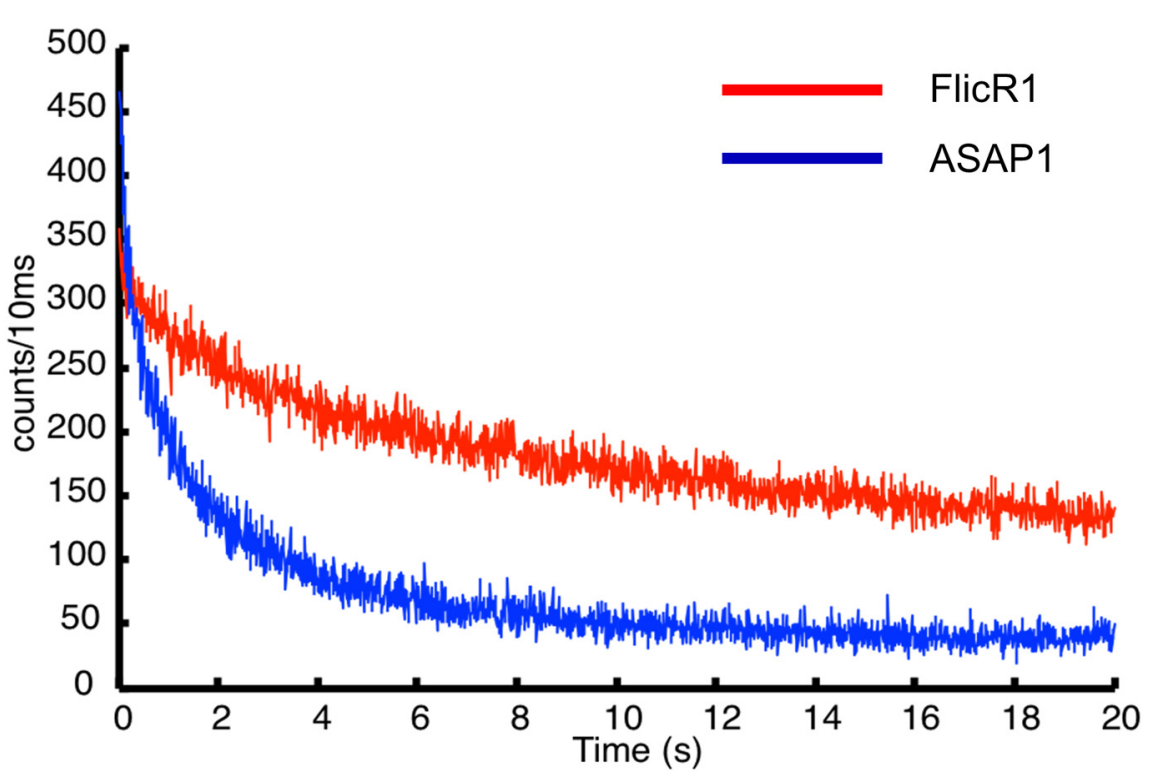

Figure 4. Two-photon imaging of FlicR1 in HEK cells. $A$, Uncorrected fluorescence response of FlicR1 during -100 to $+100 \mathrm{mV}$ voltage steps $(0.25 \mathrm{~Hz}$ square wave, $2 \mathrm{~s}+100 \mathrm{mV}, 2 \mathrm{~s}-100 \mathrm{mV}$ ) when excited at $1120 \mathrm{~nm}$ in image-scanning mode $(6.5 \mathrm{frames} / \mathrm{s})$. $\boldsymbol{B}$, Fluorescence intensity as a function of time for a $1000 \mathrm{~Hz}$ point scan of FlicR1 fluorescence during a $100 \mathrm{~Hz}$ voltage square wave $(5 \mathrm{~ms}+100 \mathrm{mV}$ FlicR1 excited at $1120 \mathrm{~nm}$. Fluorescence is sampled at $100 \mathrm{~Hz}$, with excitation power ( $\sim 1 \mathrm{~mW}$ at both wavelengths) tuned to achieve

sis, we transfected cultured hippocampal neurons with FlicR1 driven under the control of human synapsin I promoter.

FlicR1 labeled the plasma membrane both at the cell body and in individual dendrites (Fig. 5A). It also showed intracellular puncta in the cell bodies similar to other cpmApple-derived indicators. When imaged at $100 \mathrm{~Hz}$ using a wide-field fluorescence microscope with a $200 \mathrm{~W}$ lamp $\left(0.2 \mathrm{~W} / \mathrm{cm}^{2}\right.$ power fluence at sample), membrane-localized FlicR1 clearly reported spontaneous activity of the transfected neurons (Fig. $5 B, C$ ) with a signal amplitude of $\Delta F / F=4.3 \pm 0.6 \%(n=7)$ corresponding to an SNR of $9 \pm 2(n=7)$.

We next characterized the response of FlicR1 to action potentials in neurons. All measurements were performed at $22^{\circ} \mathrm{C}$ at a frame rate of $1 \mathrm{kHz}$ while illuminating with $10 \mathrm{~W} / \mathrm{cm}^{2} 561 \mathrm{~nm}$ light. In whole-cell current-clamp mode, we stimulated action potentials in neurons transfected with either FlicR1 or ArcLight Q293. FlicR1 faithfully reported single action potentials with a change in fluorescence of $\Delta F / F=2.6 \pm 0.8 \%(n=7)$, averaging over the entire cell 

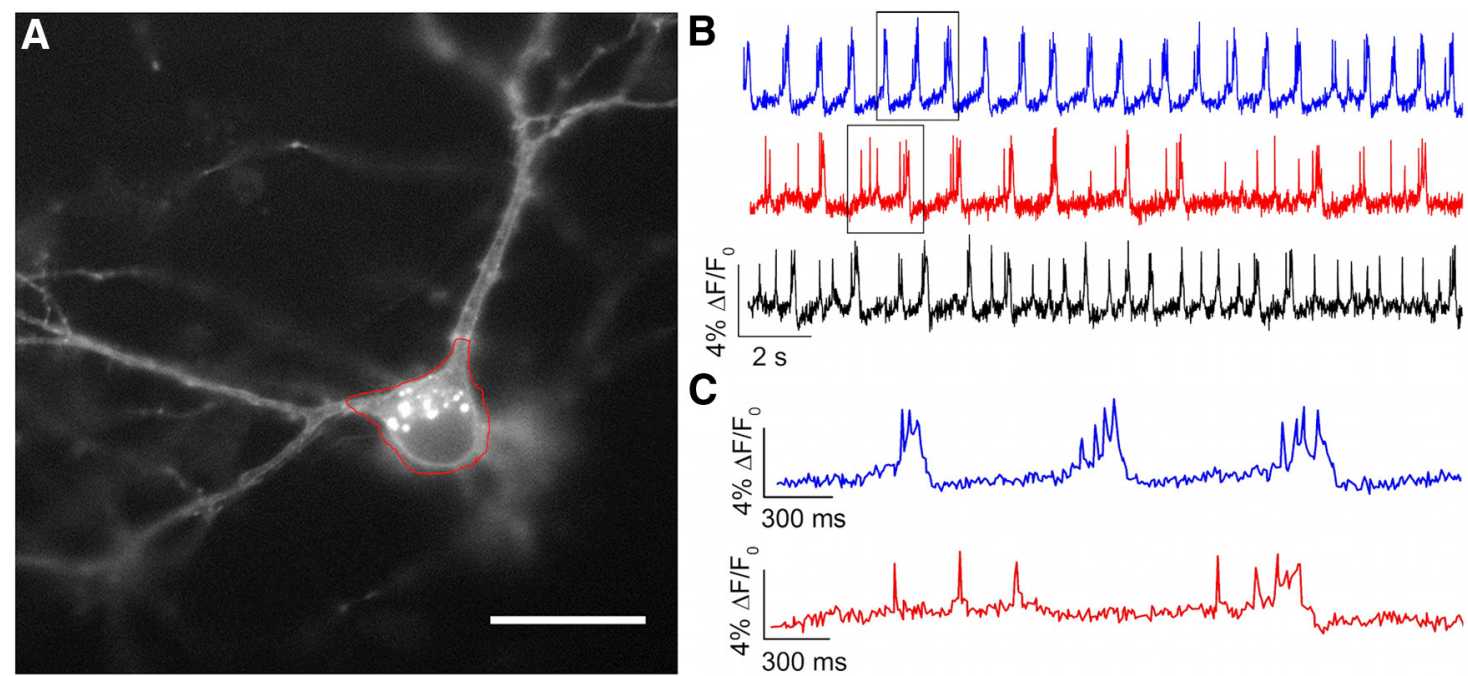

D

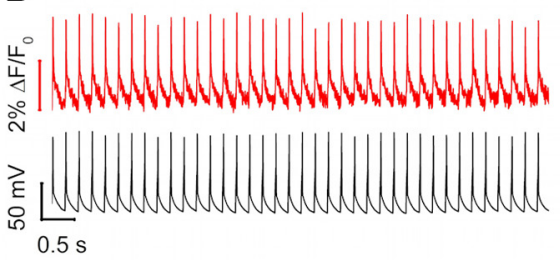

E
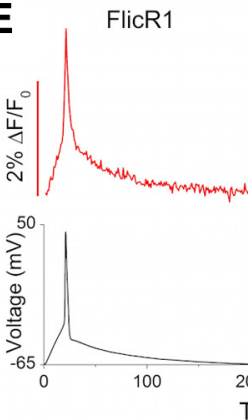

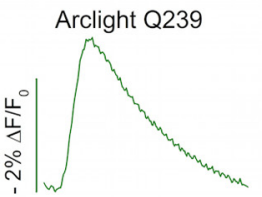

50

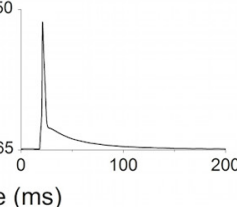

$\mathbf{F}$

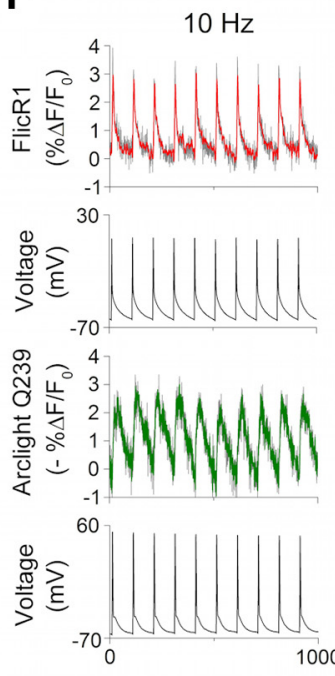

$50 \mathrm{~Hz}$
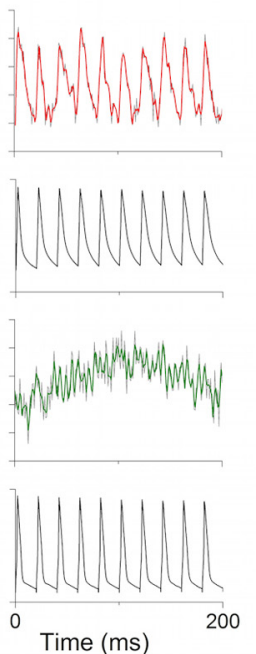

$100 \mathrm{~Hz}$
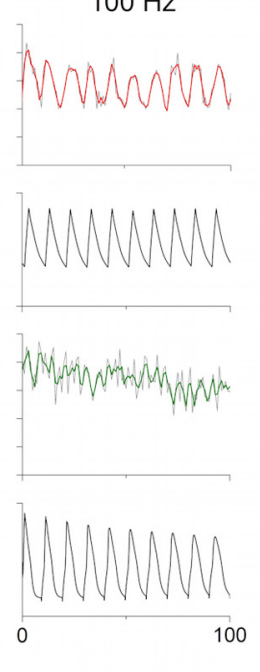

Figure 5. FlicR1 characterization in neurons. $A$, Image of cultured hippocampal neuron expressing FlicR1. Scale bar, $30 \mu \mathrm{m}$. $\boldsymbol{B}$, Detection of spontaneous activity waveforms in rat hippocampal neuron culture with FlicR1 indicator. Sample single-trial recordings of spontaneous action potential bursts from three neurons. Red trace is from cell in $\boldsymbol{A}$. C, Magnification of regions marked in $\boldsymbol{B}$. D, FlicR1 fluorescence response to $5 \mathrm{~Hz}$ stimulated action potential train. $\boldsymbol{E}$, Mean fluorescence response of FlicR1 (left) and Arclight Q239 (right). F, FlicR1 (top) and Arclight (bottom) response to 10, 50 , and $100 \mathrm{~Hz}$ stimulated action potential trains in neurons. Colored traces are filtered with Savitzky-Golay smoothing (5 points) and are overlaid over the grayscale unfiltered traces. All traces have single exponential correction of bleach. For $\boldsymbol{A}-\boldsymbol{C}$, fluorescence was recorded at $100 \mathrm{~Hz}$ frame rate and illumination intensity was $0.2 \mathrm{~W} / \mathrm{cm}^{2}$. For $\boldsymbol{D}-\boldsymbol{F}$, fluorescence was acquired at a $1 \mathrm{kHz}$ frame rate and $10 \mathrm{~W} / \mathrm{cm}^{2}$ illumination intensity. Colored traces in $\boldsymbol{D}-\boldsymbol{F}$ are filtered with Savitzky-Golay smoothing (5 points) for both FlicR1 and Arclight Q239.

body (Fig. 5D), corresponding to an SNR of $6 \pm 1(n=7)$. ArcLight Q239 showed a similar fluorescence response amplitude to single action potentials $(\triangle F / F=-3.4 \pm 0.9 \%, n=5)$. However, because FlicR1 had faster kinetics than ArcLight, the fluorescence signal of FlicR1 more closely matched the electrical trace and was not broadened to the extent that it was with ArcLight Q239 (Fig. 5E). To investigate FlicRl's ability to track high-frequency firing in neurons, we stimulated action potentials at frequencies from $5 \mathrm{~Hz}$ to $100 \mathrm{~Hz}$ $(n=7)$. FlicR1 reported voltage fluctuations of up to $100 \mathrm{~Hz}$, clearly discriminating individual peaks in single trial recordings (Fig. 5F). In contrast, ArcLight Q239 was not able to discriminate peaks at speeds $>10 \mathrm{~Hz}$ and only showed increased baseline fluorescence (Fig. $5 F$ ). We detected no significant differences in action potential amplitude ( $p=0.16$, two-tailed Student's $t$ test), membrane resistance $(p=0.67)$, or membrane capacitance $(p=0.21)$ between ArcLight Q239 $(n=6)$ and FlicR1 $(n=7)$ transfected cells.

\section{Imaging brain slice activity with FlicR1}

To test whether FlicR1 provides sufficient spatial and temporal resolution to report spontaneous activity in neuronal tissue, we expressed and imaged FlicR1 in organotypic rat brain slices. Organotypic rat hippocampal slices have been shown to maintain native hippocampal morphology and function and show spontaneous network activity (De Simoni et al., 2003). FlicR1 was delivered under human synapsin I promoter using ex vivo electroporation. To test the long-term effect of expression of FlicR1, hippocampal slices were cultured for 22-25 d (18-21 d after transfection). Transfected neurons were clearly visible under wide-field epifluorescence imaging (Fig. 6A,D). Wide-field epifluorescence imaging of FlicR1 successfully reported spontaneous activity of transfected neurons in single-trial optical recordings when excited with constant yellow light illumination using a $100 \mathrm{~W}$ mercury lamp $\left(0.4 \mathrm{~W} / \mathrm{cm}^{2}\right.$ power fluence at sample). A signal amplitude of $\Delta F / F=4 \pm 1 \%$ and an 

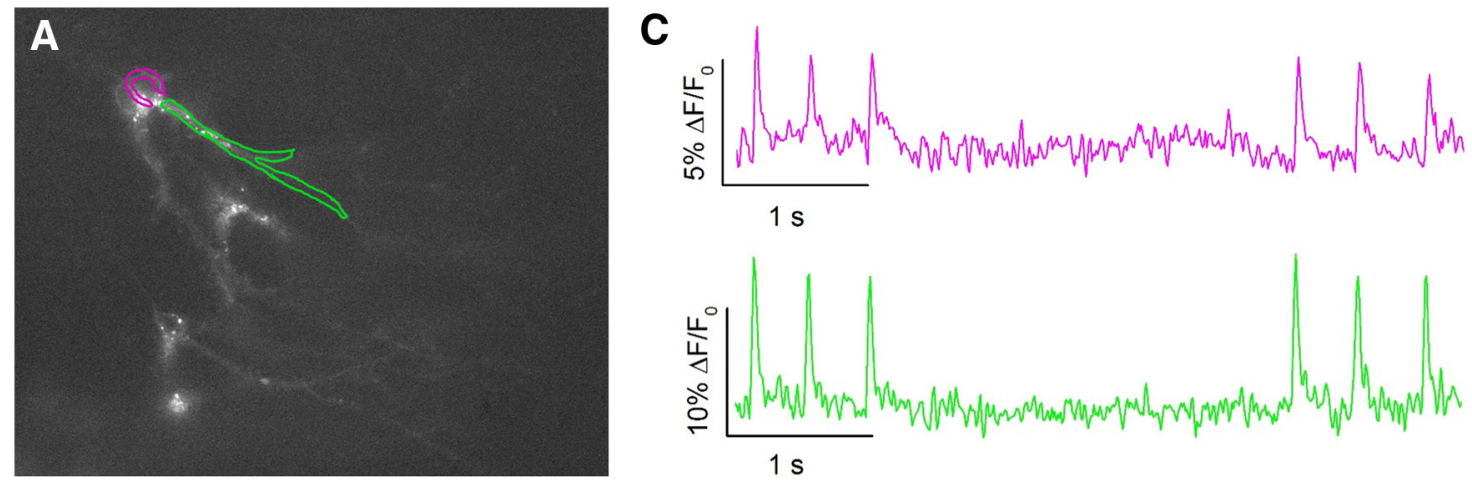

B
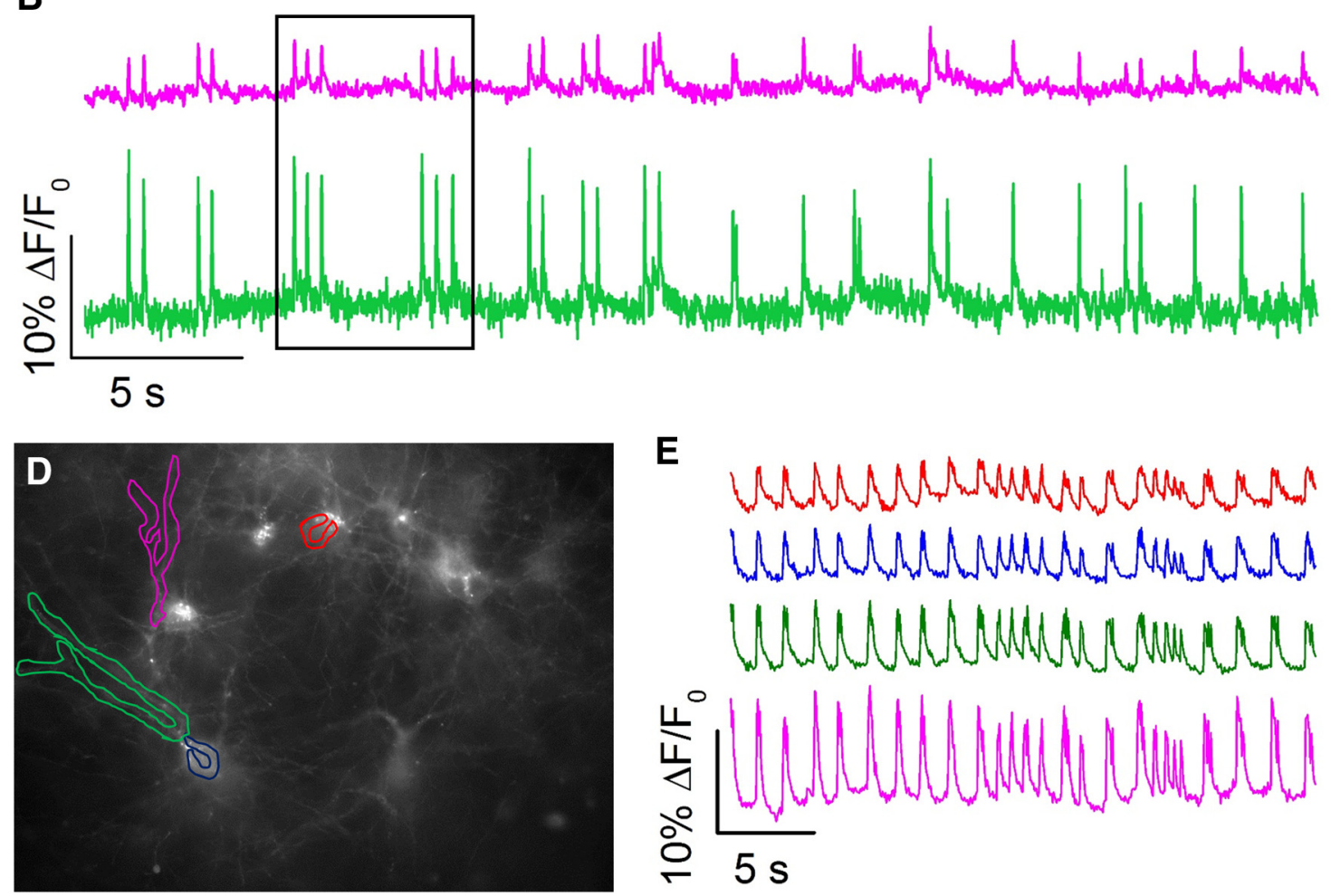

Figure 6. Detection of spontaneous activity and theophylline-induced activity in rat hippocampal brain slice with FlicR1 indicator. A, Fluorescence image of hippocampal brain slice transfected with FlicR1 and imaged $21 \mathrm{~d}$ after transfection. Neuron processes are clearly labeled with FlicR1. Both cell bodies and processes show some fluorescence puncta. $\boldsymbol{B}$, Single-trial fluorescence traces of activity in neuron cell bodies and neuron processes imaged with FlicR1. The traces correspond to the regions in the image marked with the same color. These traces are from wide-field fluorescence data acquired using a $100 \mathrm{~W}$ mercury lamp at $100 \mathrm{~Hz}$ imaging frequency. $\boldsymbol{C}$, Magnification of traces in $\boldsymbol{B}$ marked with black borderline. $\boldsymbol{D}$, Fluorescence image of hippocampal brain slice transfected with FlicR1 and imaged $18 \mathrm{~d}$ after transfection. Neuron processes are clearly labeled with FlicR1. Both cell bodies and processes show some fluorescent puncta. E, Fluorescence traces acquired at 50 $\mathrm{Hz}$ of theophylline-induced membrane depolarization in neuron cell bodies and neuron processes imaged with FlicR1. The traces correspond to the regions in the image marked with the same color. All fluorescence traces are bleach corrected and traces in $\boldsymbol{B}$ and $\boldsymbol{C}$ are filtered with Savitzky-Golay smoothing (5 pts). Fluorescence traces were recorded at $100 \mathrm{~Hz}(\boldsymbol{A}-\boldsymbol{C})$ and $50 \mathrm{~Hz}(\boldsymbol{D}, \boldsymbol{E})$ frame rate. Illumination intensity was $0.4 \mathrm{~W} / \mathrm{cm}^{2}$.

SNR of $8 \pm 2(n=12$ cells) was obtained when averaging over the whole cell body. This was compared with a signal amplitude of $\Delta F /$ $F=9 \pm 2 \%(n=12$ cells $)$ and an SNR of $8 \pm 2$ obtained by averaging multiple ROIs in dendritic regions (Fig. $6 B, C$ ). We attribute the difference in signal amplitude to the presence of unresponsive puncta in the cell bodies. We also used theophylline to pharmacologically excite FlicR1-transfected neurons in organotypic brain slices. Theophylline has been shown to evoke sustained rhythmic, seizure-like activities in different neuronal networks including hippocampal neurons (Panaitescu et al., 2013). Indeed, upon treatment with theophylline, FlicR1 successfully reported seizure-like voltage oscillations when imaged at $50 \mathrm{~Hz}$ using the same $100 \mathrm{~W}$ mercury lamp with a signal amplitude of $\Delta F / F=9 \pm 2 \%(n=12$ cells $)$, which corresponds to an SNR of $14 \pm 2(n=12)$ (Fig. $6 E$ ).

\section{All optical electrophysiology}

Next, we explored the utility of FlicR 1 as a voltage indicator in an all-optical electrophysiology setup. One advantage of a red voltage indicator is its orthogonal spectrum to blue-light-excitable channelrhodopsin actuators, suggesting a pairing of FlicR1 with a blue-light excitable channelrhodopsin for all-optical electrophysiology. As a proof-of-concept experiment, we cotransfected HeLa cells with FlicR1 and the channel rhodopsin variant ChIEF (Lin et al., 2009). Both FlicR1 and ChIEF showed high expression and good membrane trafficking when expressed in HeLa cells (Fig. 7A,B). FlicR1 successfully reported blue light (405 nm, 20 $\mathrm{mW} / \mathrm{cm}^{2}$ )-stimulated depolarization of HeLa cells with a fluorescence change of $\Delta F / F=6.1 \pm 0.7 \%(n=8)$ (Fig. $7 D, E)$. As expected, at the image acquisition rate of $100 \mathrm{~Hz}$, FlicR1 did not 

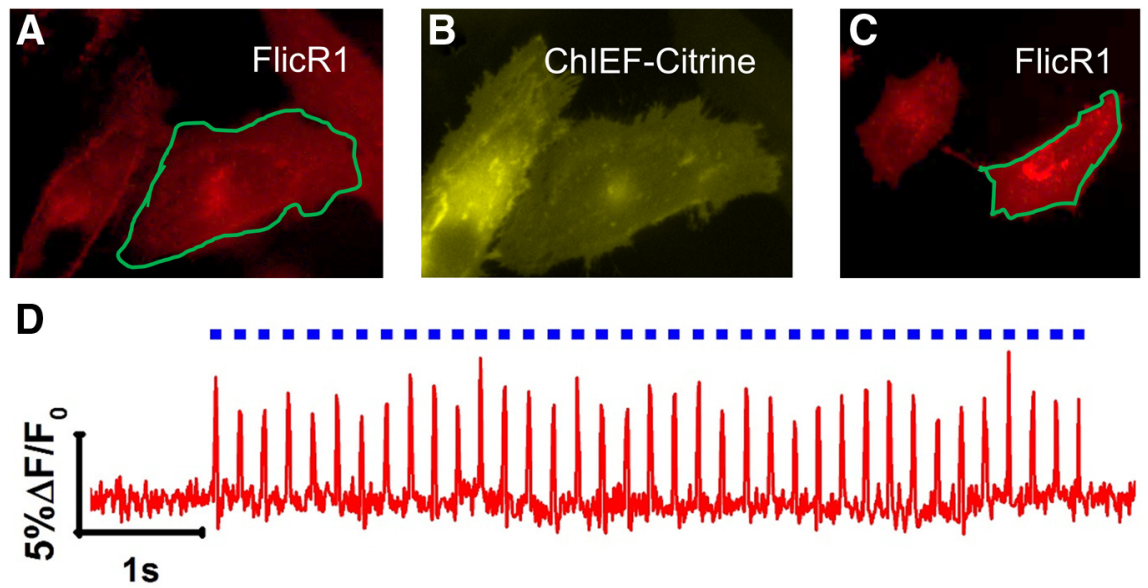

E

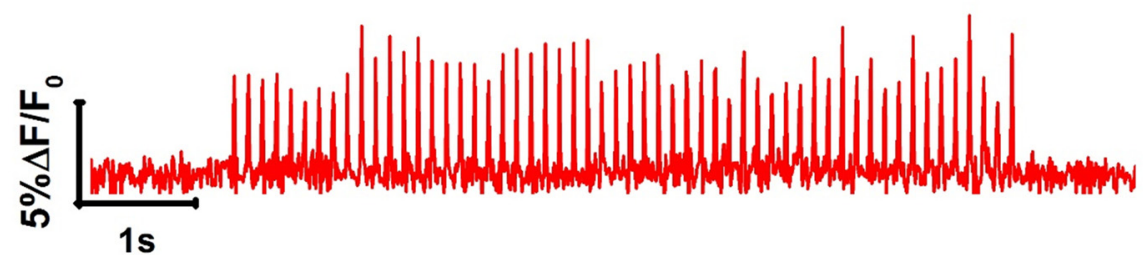

$\mathbf{F}$
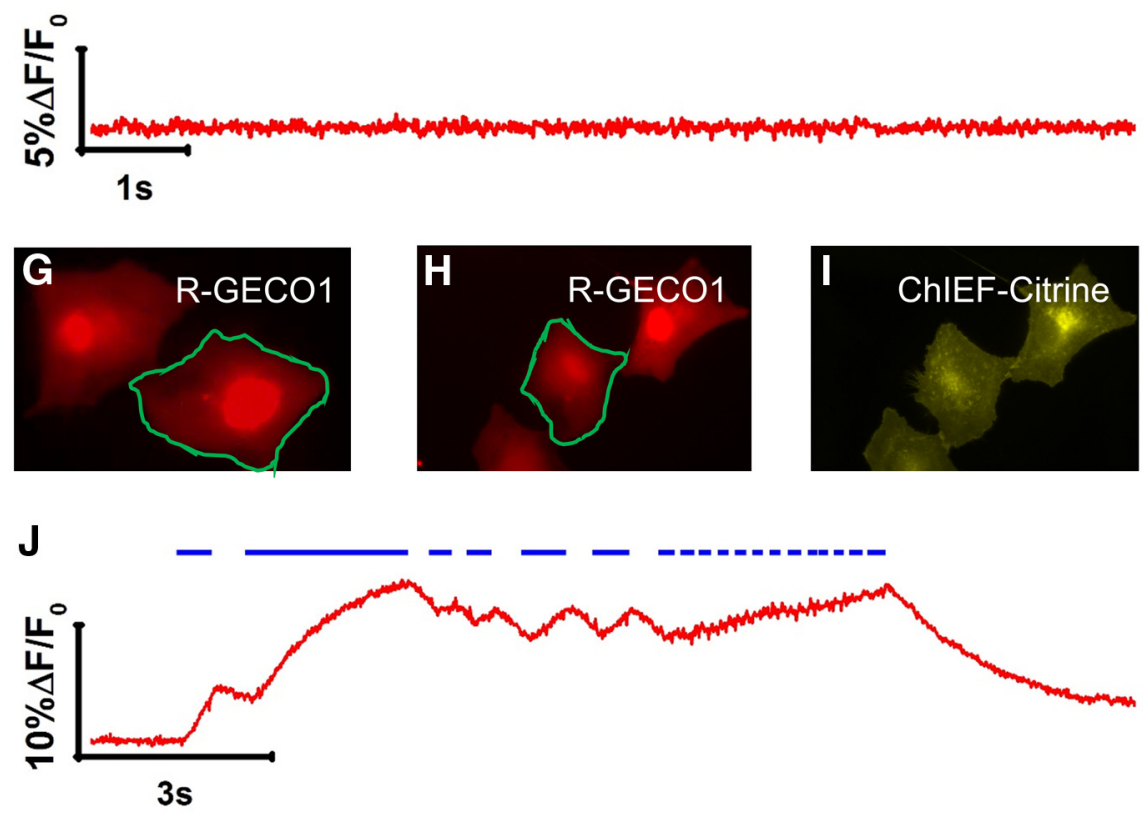

K

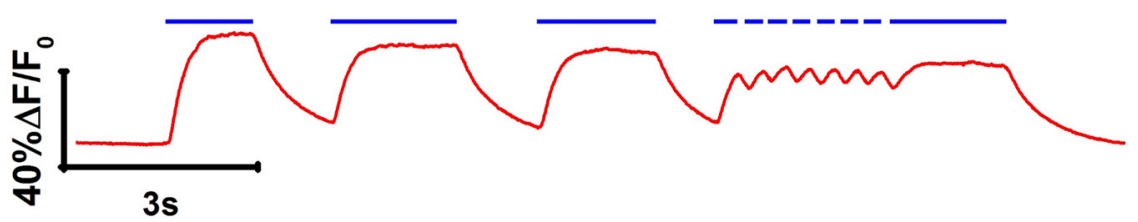

Figure 7. All optical electrophysiology with FlicR1 indicator in mammalian cells and comparison with R-GEC01 photoactivation. $\boldsymbol{A}, \boldsymbol{B}$, Image of HeLa cells coexpressing FlicR1 $(\boldsymbol{A})$ and ChIEF-Citrine $(\boldsymbol{B})$. C, Image of HeLa cells expressing FlicR1 only. $\boldsymbol{D}$, Fluorescence trace of FlicR 1 in HeLa cells activated by $5 \mathrm{~Hz}$ stimulation of ChIEF using $405 \mathrm{~nm}$ laser pulses. Shown is a trace from the cell in $\boldsymbol{A}$. $\boldsymbol{E}$, Fluorescence trace of FlicR1 in HeLa cells activated by $10 \mathrm{~Hz}$ stimulation of ChIEF using $405 \mathrm{~nm}$ laser pulses. Shown is a trace from the cell in $\boldsymbol{A}$. $\boldsymbol{F}$, Control fluorescence trace for FlicR1 in HeLa cells using $405 \mathrm{~nm}$ laser pulses without coexpression of ChIEF. Shown is a trace from the cell in $\mathbf{C}$. $\mathbf{G}$, Image of HeLa cells expressing R-GECO1 only. $\boldsymbol{H}, \boldsymbol{I}$, Image of HeLa cells coexpressing R-GECO1 $(\boldsymbol{H})$ and ChIEF-Citrine (I). $\boldsymbol{J}$, Control fluorescence trace for R-GECO1 in HeLa cells using $405 \mathrm{~nm}$ laser pulses. Shown is a trace from the cell in show any delay in response to the blue light stimulation of ChIEF. Although FlicR1 is based on mApple, the same FP backbone as the R-GECO1 $\mathrm{Ca}^{2+}$ indicator, which shows blue light photoactivation (Wu et al., 2013; Fig. 7G-K), control cells transfected with FlicR1 alone showed no photoactivation with the $20 \mathrm{~mW} / \mathrm{cm}^{2}$ $405 \mathrm{~nm}$ blue light used to stimulate HeLa cells (Fig. 7C,F)

Although we were encouraged by these results, the $405 \mathrm{~nm}$ light intensity in our proof-of-concept experiment was 10-fold smaller than the light intensities necessary to induce action potentials reliably in cultured hippocampal neurons with most commonly used channelrhodopsin actuators (Mattis et al., 2012). We therefore characterized the response of FlicR1 under blue light illumination conditions comparable to those necessary for neuronal experiments. We transfected HEK cells with FlicR1 and monitored their fluorescence with a $100 \mathrm{~Hz}$ imaging rate under $10 \mathrm{~W} / \mathrm{cm}^{2} 561 \mathrm{~nm}$ illumination while using whole-cell patch clamp to vary the membrane voltage for three cycles between -100 to $+50 \mathrm{mV}$. For the third cycle, we also illuminated the cell with 208 $\mathrm{mW} / \mathrm{cm}^{2} 458 \mathrm{~nm}$ light. The fluorescence traces showed significant photoactivation of FlicR 1 by the blue light and a simultaneous decrease in voltage sensitivity. We also repeated the measurement with 25 $\mathrm{mW} / \mathrm{cm}^{2} 405 \mathrm{~nm}$ light and observed a similar photoactivation effect in one of four runs. These results indicated that, under the blue light illumination conditions necessary to drive action potentials with channelrhodopsin actuators, photoactivation of FlicR1 could still present a challenge. Nevertheless, we attempted to use FlicR1 for all-optical electrophysiology in neurons. We cotransfected cultured rat hippocampal neurons with FlicR1 and TsChR, a blue-shifted channelrhodopsin (Klapoetke et al., 2014), and attempted to elicit action potentials with blue light illumination while monitoring FlicR1 fluorescence. We found that illumination pulses of $10-1000 \mathrm{~ms}$ at $5-210$ $\mathrm{mW} / \mathrm{cm}^{2}$ for both 458 and $488 \mathrm{~nm}$ light (data not shown) caused sufficient photoactivation of FlicR1 to obscure its voltage response. This is in contrast to HeLa cells,

$\leftarrow$

G. $\boldsymbol{K}$, Fluorescence trace of R-GECO1 in HeLa cells activated by stimulation of ChIEF using $405 \mathrm{~nm}$ laser pulses. Shown is a trace from the cell in $\boldsymbol{H}$. Fluorescence traces were recorded at $100 \mathrm{~Hz}$. The intensity of yellow light used to image FlicR1 and R-GEC01 was $60 \mathrm{~mW} / \mathrm{cm}^{2} .405 \mathrm{~nm}$ laser intensity to activate ChIEF was $20 \mathrm{~mW} / \mathrm{cm}^{2}$ in all experiments. 
A

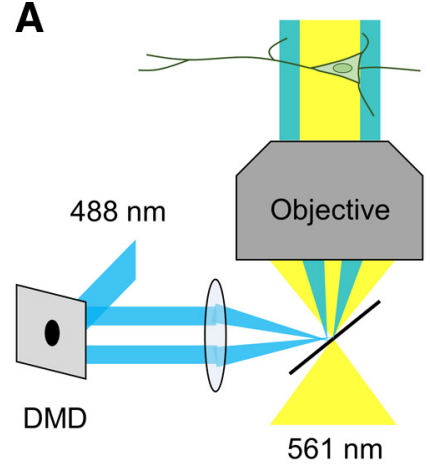

C

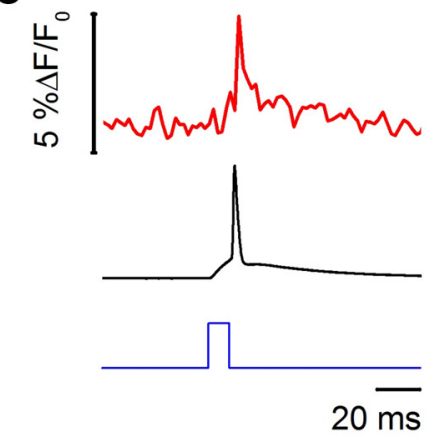

D

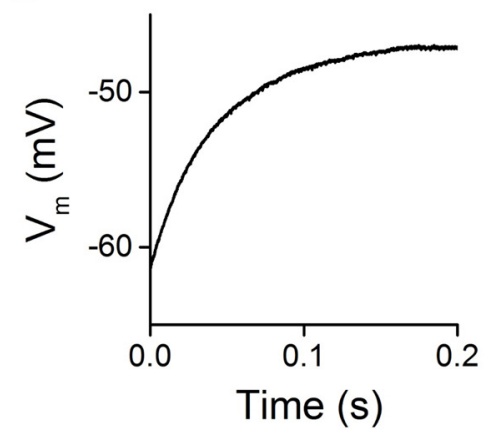

B

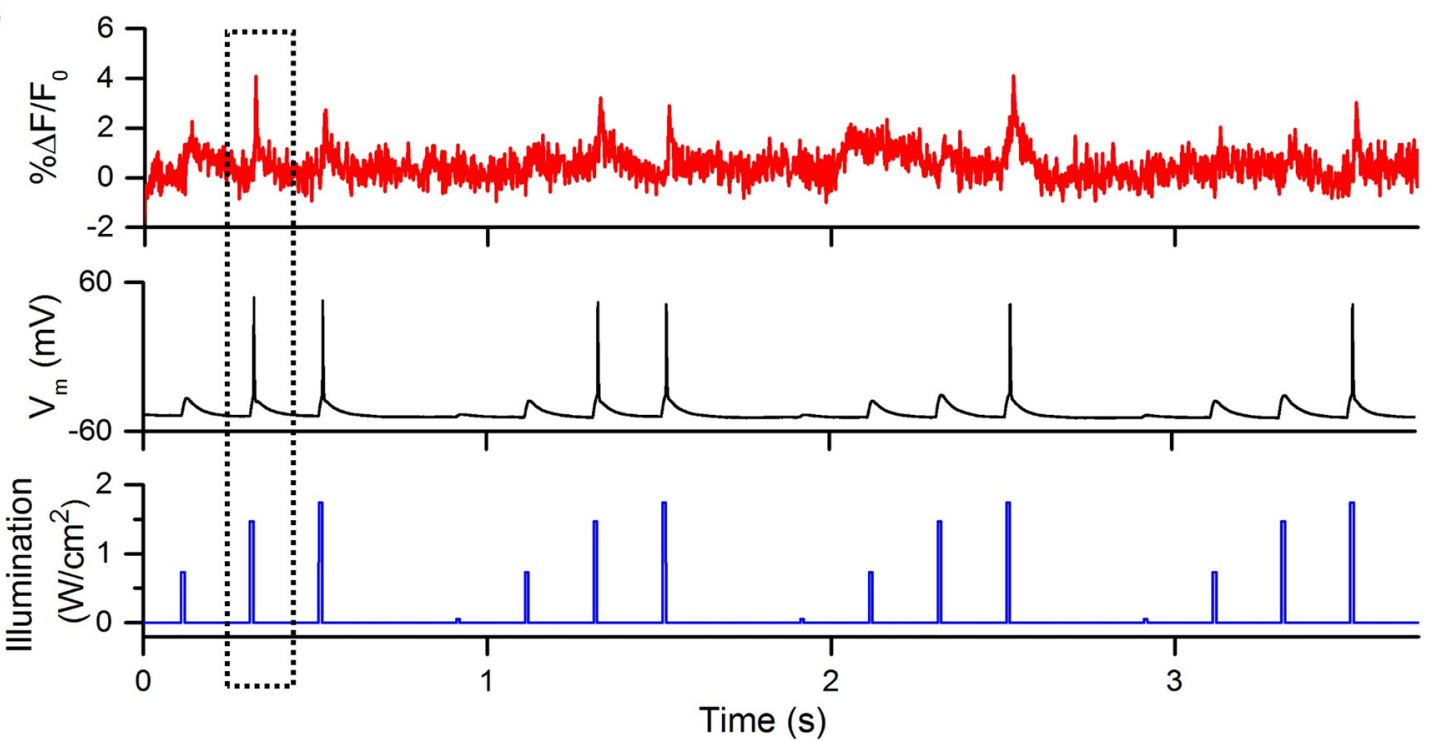

Figure 8. All-optical electrophysiology using FlicR1 in cultured hippocampal neurons. $A$, Diagram showing experimental setup using a digital micromirror device (DMD) to target the blue light to the neuronal processes. $\boldsymbol{B}$, Red, FlicR1 fluorescence readout from single-trial optical recording of single action potentials initiated by pulses of blue light illumination using the experimental setup shown in $\boldsymbol{A}$. Yellow illumination to image FlicR1 was $10 \mathrm{~W} / \mathrm{cm}^{2}$. Black, Patch-clamp recording. Blue, $488 \mathrm{~nm}$ illumination $\left(10 \mathrm{~ms}, 0.5-2 \mathrm{~W} / \mathrm{cm}^{2}\right)$. C, Magnification of traces in $\boldsymbol{B}$ marked with black borderline. $\boldsymbol{D}$, Patch-clamp recording of neuron expressing PsChR when exposed to $561 \mathrm{~nm}$ laser $\left(10 \mathrm{~W} / \mathrm{cm}^{2}\right)$. This illumination depolarized the cell by $14 \mathrm{mV}$, but did not induce action potentials on its own. All fluorescence traces are bleach corrected. Fluorescence trace was collected at a frame rate of $500 \mathrm{~Hz}$ using an EMCCD camera. Fluorescence trace in C is filtered with Savitzky-Golay smoothing (5 points).

in which $20 \mathrm{~mW} / \mathrm{cm}^{2}$ of $405 \mathrm{~nm}$ light did not cause photoactivation. We suspect that the difference between neurons and HeLa cells is attributable to intracellular puncta that are present in neurons but absent in HeLa cells. These puncta represent a large pool of protein that does not contribute to the voltage-sensitive response yet can undergo photoactivation.

To circumvent photoactivation of FlicR1 in neurons, we used a digital micromirror device (Fig. $8 A$ ) to target spatially $488 \mathrm{~nm}$ illumination pulses $\left(5 \mathrm{~mW} / \mathrm{cm}^{2}\right.$ to $1.7 \mathrm{~W} / \mathrm{cm}^{2}, 10$ or $50 \mathrm{~ms}$ duration) to the neuronal processes of cells coexpressing FlicR1 and either ChR2H134R, TsChR, or PsChR (Govorunova et al., 2013), another blueshifted channelrhodopsin, while avoiding blue illumination of the soma. We illuminated the whole cell with $561 \mathrm{~nm}$ light at $10 \mathrm{~W} / \mathrm{cm}^{2}$ and collected FlicR1 fluorescence from the soma. We used simultaneous manual patch clamp in current-clamp mode to provide independent detection of action potentials. When blue illumination was excluded from the soma, cells coexpressing FlicR1 and ChR2H134R did not produce sufficient photocurrents to induce action potentials under any illumination parameters, consistent with previous reports (Hochbaum et al., 2014). In a neuron expressing both FlicR1 and PsChR, FlicR1 could report optically-induced action potentials with a similar SNR as electrically induced action potentials
(Fig. 8B,C). The $561 \mathrm{~nm}$ illumination used for eliciting FlicR1 fluorescence depolarized the cell by $14 \mathrm{mV}$ (Fig. $8 D$ ), but did not induce action potentials on its own. This result suggests that FlicR1 could be used for all-optical electrophysiology under the appropriate expression conditions.

\section{Discussion}

Four design strategies have been used to engineer genetically encoded voltage indicators. The first uses a voltage-sensitive domain fused to an FP (Siegel and Isacoff, 1997; Ataka and Pieribone, 2002; Lundby et al., 2008; Gautam et al., 2009; Perron et al., 2009; Barnett et al., 2012; Jin et al., 2012; Han et al., 2013; St-Pierre et al., 2014). In this class, two green FP-based voltage indicators, ArcLight (Jin et al., 2012) and ASAP1 (St-Pierre et al., 2014), are able to resolve single action potentials in neurons in single trials. ArcLight has the highest response amplitude ( $~ 30 \%$ $\Delta F / F$ to a $100 \mathrm{mV}$ step; Jin et al., 2012) yet reported for an FPbased voltage indicator. However, its slow kinetics produces a response to action potentials of only $\sim 3 \% \Delta F / F$ (Jin et al., 2012). The ASAP1 indicator has a smaller response amplitude $(\sim 17 \%$ to a $100 \mathrm{mV}$ step; St-Pierre et al., 2014), but exhibits faster kinetics, 
and therefore its response to action potentials is $\sim 5 \% \Delta F / F$ (StPierre et al., 2014).

The second class of voltage indicators also uses a voltagesensitive domain, but relies on the modulation of Förster resonance energy transfer (FRET) between two fused FPs (Akemann et al., 2012; Lam et al., 2012). Although these sensors can have relatively large ratiometric changes $(>10 \%)$, the kinetics are typically slow ( $\tau>10 \mathrm{~ms}$; Akemann et al., 2012; Lam et al., 2012). The third class is based on microbial rhodopsins, which can exhibit voltage-dependent changes in the fluorescence of their bound retinal cofactor (Kralj et al., 2011, 2012). Although these indicators show larger response to voltage changes, the brightest variants, known as QuasArs (Hochbaum et al., 2014) are still much dimmer than FP-based voltage indicators (Gong et al., 2013; Hochbaum et al., 2014). One important advantage of the microbial rhodopsin-based indicators is that, relative to the currently preferred members of the other classes, they have a nearinfrared fluorescent emission. The fourth class is based on FRET from a FP to a microbial rhodopsin with a voltage-dependent absorption (Gong et al., 2014; Zou et al., 2014). This design circumvents the brightness issue of the third class at the cost of a blue shift in fluorescence emission and slower kinetics (Gong et al., 2014; Zou et al., 2014). Although many of the previously reported FP-based voltage indicators have one or more nearideal properties, none has yet emerged as the optimal combination of all favorable properties.

FlicR1 is a red indicator with performance comparable to state-of-the-art green voltage indicators such as ASAP1 and ArcLight. FlicR1 was designed with the rationale that fusion of a cpRFP to a VSD would maximize the chance of coupling voltageinduced conformational changes to the FP chromophore, leading to a large response amplitude and fast kinetics. Indeed, the large and fast response of FlicR1 indicates that the S4 movement rapidly changes the environment around the mApple chromophore. In contrast to ASAP1, FlicR1 has the cpFP linked to the end of the S4 helix. Fusion to the end of the S4 helix bestows FlicR1 with a fluorescence voltage response that is dominated by a fast component ( $\sim 3 \mathrm{~ms}$ ) for both on and off kinetics ( $90 \%$ on and $78 \%$ off) at room temperature. Although ASAP1 has slightly faster kinetics ( $\sim 2 \mathrm{~ms}$; St-Pierre et al., 2014), the fast component of the FlicR1 response has $\sim 1.5$-fold and $\sim 2$-fold increased weight for on and off, respectively, compared with ASAP1 (60\% on and $43 \%$ off). The comparable responses to single action potentials for both ASAP1 and FlicR1 is consistent with this increased weight of the fast component, because a greater fraction of the full FlicR1 response amplitude is used to report neuronal activity.

Our screening strategy was crucial to engineer FlicR1 to be a fast, bright voltage indicator with large response amplitude. Simply fusing the cpRFP resulted in a dim indicator that had a small response amplitude to voltage changes. Other reports of manually inserting cpFPs to the $\mathrm{C}$ terminus of CiVSD resulted in indicators with low response amplitude (Gautam et al., 2009; Barnett et al., 2012) and/or slow kinetics (Gautam et al., 2009). Only through the use of our screening strategy did we discover variants that are both bright and highly responsive to membrane potential changes.

One of the most intriguing mutations that resulted from random mutagenesis is Val207Ala, located in the S3 to S4 linker of the CiVSD. Subsequent randomization of this position led to the discovery of the further beneficial Val207Phe substitution. We initially reasoned that this mutation must be important for modulating the voltage sensitivity of FlicR1. The recently reported $\mathrm{x}$-ray crystal structures of CiVSD in both the activated and resting state (Li et al., 2014) show that this residue is part of an $\alpha$ helix that unwinds when the VSD is in the activated (depolarized) state. Based on this insight, we suggest that this mutation might be increasing the rate and/or extent with which the $S 4$ helix moves, thus improving FlicR1's response to membrane depolarization.

We showed that FlicR1 traffics well to the cell membrane in neurons and is suitable for long-term expression in organotypic slices. However, some fraction of the protein resides in intracellular puncta when expressed in neurons. We believe that these puncta are due to the anthozoa-derived mApple FP body of FlicR1 because related FPs often form intracellular puncta in neurons (Cai et al., 2013). These puncta, however, did not interfere with long-term expression of FlicR1 in organotypic slices. After 3 weeks of FlicR1 expression, neurons in organotypic slices showed spontaneous activity and responded to stimulation as expected. FlicR1 was $\sim 30$-fold brighter compared with archaerhodopsin-based voltage indicators, requiring only $\sim 10 \mathrm{~W} / \mathrm{cm}^{2}$ to image neural activity compared with $\sim 300$ $\mathrm{W} / \mathrm{cm}^{2}$ for the recently reported QuasAr2 (Hochbaum et al., 2014).

In conclusion, FlicRl's fast responses to single action potentials and ability to track high-frequency trains makes it an excellent sensor for action potential detection in rapid firing events. FlicR1's red fluorescence allows for all optical electrophysiology together with blue-light-excitable channelrhodopsins under illumination conditions carefully tuned to minimize optical crosstalk. Finally, FlicR1 is likely to be useful in combination with a wide variety of green fluorescent indicators, although this has not yet been demonstrated and will require crosstalk mitigation depending on the experimental setup and green indicator used.

\section{Notes}

Supplemental material for this article is available at http://campbellweb. chem.ualberta.ca. Supplemental material consists of six supporting figures. This material has not been peer reviewed.

\section{References}

Akemann W, Mutoh H, Perron A, Park YK, Iwamoto Y, Knöpfel T (2012) Imaging neural circuit dynamics with a voltage-sensitive fluorescent protein. J Neurophysiol 108:2323-2337. CrossRef Medline

Akerboom J, Carreras Calderón N, Tian L, Wabnig S, Prigge M, Tolö J, Gordus A, Orger MB, Severi KE, Macklin JJ, Patel R, Pulver SR, Wardill TJ, Fischer E, Schüler C, Chen TW, Sarkisyan KS, Marvin JS, Bargmann CI, Kim DS, et al. (2013) Genetically encoded calcium indicators for multi-color neural activity imaging and combination with optogenetics. Front Mol Neurosci 6:2. Medline

Alford SC, Wu J, Zhao Y, Campbell RE, Knöpfel T (2013) Optogenetic reporters. Biol Cell 105:14-29. CrossRef Medline

Ataka K, Pieribone VA (2002) A genetically targetable fluorescent probe of channel gating with rapid kinetics. Biophys J 82:509-516. CrossRef Medline

Baird GS, Zacharias DA, Tsien RY (1999) Circular permutation and receptor insertion within green fluorescent proteins. Proc Natl Acad Sci U S A 96:11241-11246. CrossRef Medline

Banker G, Goslin K (1998) Culturing nerve cells, Ed 2. Cambridge, MA: MIT.

Barnett L, Platisa J, Popovic M, Pieribone VA, Hughes T (2012) A fluorescent, genetically-encoded voltage probe capable of resolving action potentials. PLoS One 7:e43454. CrossRef Medline

Brinks D, Klein AJ, Cohen AE (2015) Two-photon lifetime imaging of voltage indicating proteins as a probe of absolute membrane voltage. Biophys J 109:914-921. CrossRef Medline

Brown JE, Cohen LB, De Weer P, Pinto LH, Ross WN, Salzberg BM (1975) Rapid changes in intracellular free calcium concentration: detection by metallochromic indicator dyes in squid giant axon. Biophys J 15:1155-1160. CrossRef Medline

Cai D, Cohen KB, Luo T, Lichtman JW, Sanes JR (2013) Improved tools for the Brainbow toolbox. Nat Methods 10:540-547. CrossRef Medline

Chen TW, Wardill TJ, Sun Y, Pulver SR, Renninger SL, Baohan A, Schreiter ER, Kerr RA, Orger MB, Jayaraman V, Looger LL, Svoboda K, Kim DS (2013) Ultrasensitive fluorescent proteins for imaging neuronal activity. Nature 499:295-300. CrossRef Medline

Cheng Z, Campbell RE (2006) Assessing the structural stability of designed beta- 
hairpin peptides in the cytoplasm of live cells. Chembiochem 7:1147-1150. CrossRef Medline

Cohen MR, Maunsell JH (2009) Attention improves performance primarily by reducing interneuronal correlations. Nat Neurosci 12: 1594-1600. CrossRef Medline

Davila HV, Salzberg BM, Cohen LB, Waggoner AS (1973) A large change in axon fluorescence that provides a promising method for measuring membrane potential. Nat New Biol 241:159-160. Medline

De Simoni A, Griesinger CB, Edwards FA (2003) Development of rat CA1 neurones in acute versus organotypic slices: role of experience in synaptic morphology and activity. J Physiol 550:135-147. CrossRef Medline

Gautam SG, Perron A, Mutoh H, Knöpfel T (2009) Exploration of fluorescent protein voltage probes based on circularly permuted fluorescent proteins. Front Neuroeng 2:14. Medline

Gong Y, Li JZ, Schnitzer MJ (2013) Enhanced archaerhodopsin fluorescent protein voltage indicators. PLoS One 8:e66959. CrossRef Medline

Gong Y, Wagner MJ, Zhong Li J, Schnitzer MJ (2014) Imaging neural spiking in brain tissue using FRET-opsin protein voltage sensors. Nat Commun 5:3674. Medline

Govorunova EG, Sineshchekov OA, Li H, Janz R, Spudich JL (2013) Characterization of a highly efficient blue-shifted channelrhodopsin from the marine alga Platymonas subcordiformis. J Biol Chem 288:29911-29922. CrossRef Medline

Han Z, Jin L, Platisa J, Cohen LB, Baker BJ, Pieribone VA (2013) Fluorescent protein voltage probes derived from ArcLight that respond to membrane voltage changes with fast kinetics. PLoS One 8:e81295. CrossRef Medline

Herculano-Houzel S (2012) The remarkable, yet not extraordinary, human brain as a scaled-up primate brain and its associated cost. Proc Natl Acad Sci U S A 109:10661-10668. CrossRef Medline

Hochbaum DR, Zhao Y, Farhi SL, Klapoetke N, Werley CA, Kapoor V, Zou P, Kralj JM, Maclaurin D, Smedemark-Margulies N, Saulnier JL, Boulting GL, Straub C, Cho YK, Melkonian M, Wong GK, Harrison DJ, Murthy VN, Sabatini BL, Boyden ES, et al. (2014) All-optical electrophysiology in mammalian neurons using engineered microbial rhodopsins. Nat Methods 11:825-833. CrossRef Medline

Jiang M, Chen G (2006) High Ca2+-phosphate transfection efficiency in low-density neuronal cultures. Nat Protoc 1:695-700. CrossRef Medline

Jin L, Han Z, Platisa J, Wooltorton JR, Cohen LB, PieriboneVA (2012) Single action potentials and subthreshold electrical events imaged in neurons with a fluorescent protein voltage probe. Neuron 75:779-785. CrossRef Medline

Katayama H, Yamamoto A, Mizushima N, Yoshimori T, Miyawaki A (2008) GFP-like proteins stably accumulate in lysosomes. Cell Struct Funct 33: 1-12. CrossRef Medline

Kirkton RD, Bursac N (2011) Engineering biosynthetic excitable tissues from unexcitable cells for electrophysiological and cell therapy studies. Nat Commun 2:300. CrossRef Medline

Klapoetke NC, Murata Y, Kim SS, Pulver SR, Birdsey-Benson A, Cho YK, Morimoto TK, Chuong AS, Carpenter EJ, Tian Z, Wang J, Xie Y, Yan Z, Zhang Y, Chow BY, Surek B, Melkonian M, Jayaraman V, ConstantinePaton M, Wong GK, et al. (2014) Independent optical excitation of distinct neural populations. Nat Methods 11:338-346. CrossRef Medline

Kralj JM, Hochbaum DR, Douglass AD, Cohen AE (2011) Electrical spiking in Escherichia coli probed with a fluorescent voltage-indicating protein. Science 333:345-348. CrossRef Medline

Kralj JM, Douglass AD, Hochbaum DR, Maclaurin D, Cohen AE (2012) Optical recording of action potentials in mammalian neurons using a microbial rhodopsin. Nat Methods 9:90-95. CrossRef Medline

Lam AJ, St-Pierre F, Gong Y, Marshall JD, Cranfill PJ, Baird MA, McKeown MR, Wiedenmann J, Davidson MW, Schnitzer MJ, Tsien RY, Lin MZ (2012) Improving FRET dynamic range with bright green and red fluorescent proteins. Nat Methods 9:1005-1012. CrossRef Medline

Lewin A, Mayer M, Chusainow J, Jacob D, Appel B (2005) Viral promoters can initiate expression of toxin genes introduced into Escherichia coli. BMC Biotechnol 5:19. CrossRef Medline

Li Q, Wanderling S, Paduch M, Medovoy D, Singharoy A, McGreevy R, VillalbaGalea CA, Hulse RE, Roux B, Schulten K, Kossiakoff A, Perozo E (2014) Structural mechanism of voltage-dependent gating in an isolated voltage-sensing domain. Nat Struct Mol Biol 21:244-252. CrossRef Medline

Lin JY, Lin MZ, Steinbach P, Tsien RY (2009) Characterization of engi- neered channelrhodopsin variants with improved properties and kinetics. Biophys J 96:1803-1814. CrossRef Medline

Lundby A, Mutoh H, Dimitrov D, Akemann W, Knöpfel T (2008) Engineering of a genetically encodable fluorescent voltage sensor exploiting fast Ci-VSP voltagesensing movements. PLoS One 3:e2514. CrossRef Medline

Mattis J, Tye KM, Ferenczi EA, Ramakrishnan C, O'Shea DJ, Prakash R, Gunaydin LA, Hyun M, Fenno LE, Gradinaru V, Yizhar O, Deisseroth K (2012) Principles for applying optogenetic tools derived from direct comparative analysis of microbial opsins. Nat Methods 9:159-172. Medline

McCarthy KD, de Vellis J (1980) Preparation of separate astroglial and oligodendroglial cell cultures from rat cerebral tissue. J Cell Biol 85:890 -902. CrossRef Medline

Miyawaki A, Llopis J, Heim R, McCaffery JM, Adams JA, Ikura M, Tsien RY (1997) Fluorescent indicators for $\mathrm{Ca} 2+$ based on green fluorescent proteins and calmodulin. Nature 388:882-887. CrossRef Medline

Mullinax RL, Wong DT, Davis HA, Padgett KA, Sorge JA (2002) Dualexpression vectors for efficient protein expression in both E. coli and mammalian cells. In: E. coli gene expression protocols (Vaillancourt PE, ed), pp 19-30. New Jersey: Humana.

Murata Y, Iwasaki H, Sasaki M, Inaba K, Okamura Y (2005) Phosphoinositide phosphatase activity coupled to an intrinsic voltage sensor. Nature 435:1239-1243. CrossRef Medline

Nagai T, Sawano A, Park ES, Miyawaki A (2001) Circularly permuted green fluorescent proteins engineered to sense $\mathrm{Ca} 2+$. Proc Natl Acad Sci U S A 98:3197-3202. CrossRef Medline

Nakai J, Ohkura M, Imoto K (2001) A high signal-to-noise Ca(2+) probe composed of a single green fluorescent protein. Nat Biotechnol 19: 137-141. CrossRef Medline

Panaitescu B, Kuribayashi J, Ruangkittisakul A, Leung V, Iizuka M, Ballanyi K (2013) Methylxanthines do not affect rhythmogenic preBötC inspiratory network activity but impair bursting of preBötC-driven motoneurons. Neuroscience 255:158-176. CrossRef Medline

Perron A, Mutoh H, Launey T, Knöpfel T (2009) Red-shifted voltage-sensitive fluorescent proteins. Chem Biol 16:1268-1277. CrossRef Medline

Pesaran B, Nelson MJ, Andersen RA (2008) Free choice activates a decision circuit between frontal and parietal cortex. Nature 453:406-409. CrossRef Medline

Shu X, Royant A, Lin MZ, Aguilera TA, Lev-Ram V, Steinbach PA, Tsien RY (2009) Mammalian expression of infrared fluorescent proteins engineered from a bacterial phytochrome. Science 324:804-807. CrossRef Medline

Siegel MS, Isacoff EY (1997) A genetically encoded optical probe of membrane voltage. Neuron 19:735-741. CrossRef Medline

St-Pierre F, Marshall JD, Yang Y, Gong Y, Schnitzer MJ, Lin MZ (2014) High-fidelity optical reporting of neuronal electrical activity with an ultrafast fluorescent voltage sensor. Nat Neurosci 17:884-889. CrossRef Medline

Tsien RY (1980) New calcium indicators and buffers with high selectivity against magnesium and protons: design, synthesis, and properties of prototype structures. Biochemistry 19:2396-2404. CrossRef Medline

Wang Q, Shui B, Kotlikoff MI, Sondermann H (2008) Structural basis for calcium sensing by GCaMP2. Structure 16:1817-1827. CrossRef Medline

Wu J, Liu L, Matsuda T, Zhao Y, Rebane A, Drobizhev M, Chang YF, Araki S, Arai Y, March K, Hughes TE, Sagou K, Miyata T, Nagai T, Li WH, Campbell RE (2013) Improved orange and red $\mathrm{Ca}^{2} \pm$ indicators and photophysical considerations for optogenetic applications. ACS Chem Neurosci 4:963-972. CrossRef Medline

Wu J, Abdelfattah AS, Miraucourt LS, Kutsarova E, Ruangkittisakul A, Zhou H, Ballanyi K, Wicks G, Drobizhev M, Rebane A, Ruthazer ES, Campbell $\mathrm{RE}$ (2014) A long Stokes shift red fluorescent $\mathrm{Ca} 2+$ indicator protein for two-photon and ratiometric imaging. Nat Commun 5:5262. CrossRef Medline

Zhao Y, Araki S, Wu J, Teramoto T, Chang YF, Nakano M, Abdelfattah AS, Fujiwara M, Ishihara T, Nagai T, Campbell RE (2011) An expanded palette of genetically encoded $\mathrm{Ca}^{2+}$ indicators. Science 333:1888-1891. CrossRef Medline

Zou P, Zhao Y, Douglass AD, Hochbaum DR, Brinks D, Werley CA, Harrison DJ, Campbell RE, Cohen AE (2014) Bright and fast multicoloured voltage reporters via electrochromic FRET. Nat Commun 5:4625. Medline 